\title{
On an optimization problem related to static super-replicating strategies
}

\author{
Xinliang Chen* Griselda Deelstra $^{\dagger} \quad$ Jan Dhaene $^{\ddagger} \quad$ Daniël Linders $^{\S}$ \\ Michèle Vanmaele
}

Version: May 12, 2014

\begin{abstract}
In this paper, we investigate an optimization problem related to super-replicating strategies for European-type call options written on a weighted sum of asset prices, following the initial approach in Chen et al. (2008). Three issues are investigated. The first issue is the (non-)uniqueness of the optimal solution. The second issue is the generalization to an optimization problem where the weights may be random. This theory is then applied to static super-replication strategies for some exotic options in a stochastic interest rate setting. The third issue is the study of the co-existence of the comonotonicity property and the martingale property.
\end{abstract}

Keywords: Asian options, basket options, comonotonicity, super-hedging strategies.

Disclaimer: The views expressed are those of the authors, and do not necessarily reflect the position of their respective employers.

${ }^{*}$ ING, Brussels, Belgium, Email: xinliang.chen@ hotmail.com

†Université Libre de Bruxelles, Brussels, Belgium, Email: griselda.deelstra@ulb.ac.be

${ }^{\ddagger}$ KU Leuven, Leuven, Belgium. Email: Jan.Dhaene@ @uleuven.be

${ }^{\S}$ KU Leuven, Leuven, Belgium. Email: Daniel.Linders@kuleuven.be

๑Ghent University, Gent, Belgium, Email: michele.vanmaele@UGent.be 


\section{Introduction}

Self-financing portfolios play an important role in hedging, trading and valuation. When a selffinancing portfolio dominates an exotic option in terms of its pay-off, it is a super-replicating portfolio. In addition, when the weights of the elements in a super-replicating portfolio are fixed from the starting time, it is a static super-replicating portfolio, and the corresponding strategy is called a static super-replicating strategy.

For a given exotic option, in general, several strategies will exist which super-replicate its pay-off. One of the aims of this paper is to investigate the problem of finding the cheapest strategy in a well-defined class of admissable super-replicating strategies for the exotic option under consideration.

\subsection{Static super-replicating strategies}

For $i=1,2, \ldots, n$, the random variable $X_{i}$, defined on the probability space $(\Omega, \mathcal{F}, \mathbb{P})$ denotes the price of an asset at some future date $T_{i}, 0 \leq T_{i} \leq T$. Hereafter, we always assume that all $X_{i}$ are positive r.v.' $\mathrm{s}^{1}$ The current time- 0 price of a European call option with pay-off $\left(X_{i}-K\right)_{+}$at maturity $T_{i}$ is denoted by $C_{i}[K]$. We assume that these options are traded on an options exchange and we can observe the market prices for these options.

Chen et al. (2008) consider a class of European call type exotic options written on $S=$ $\sum_{i=1}^{n} w_{i} X_{i}$ for some deterministic weights $w_{i}>0$, which have a pay-off at expiration time $T$ equal to $(S-K)_{+}$. The inequality

$$
(S-K)_{+}=\left(\sum_{i=1}^{n} w_{i} X_{i}-K\right)_{+} \leq \sum_{i=1}^{n} w_{i}\left(X_{i}-K_{i}\right)_{+}, \quad \mathbb{P} \text {-a.s. }
$$

always holds for all $\left(K_{1}, K_{2}, \ldots, K_{n}\right)$ satisfying $\sum_{i=1}^{n} w_{i} K_{i} \leq K$ and $K_{i} \geq 0, i=1, \ldots, n$. Static super-replicating strategies with pay-off $\sum_{i=1}^{n} w_{i}\left(X_{i}-K_{i}\right)_{+}, \sum_{i=1}^{n} w_{i} K_{i} \leq K$, are studied

\footnotetext{
${ }^{1}$ Throughout this paper, all random variables are assumed to have finite expectations.
} 
in Chen et al. (2008) in a deterministic interest rate setting.

It has been proven that one can obtain an 'optimal' decomposition $K=\sum_{i=1}^{n} w_{i} K_{i}^{*}$ with an explicit expression for the optimal $K_{i}^{*}, i=1, \ldots, n$; see Simon et al. (2000) or Dhaene et al. (2002a). A simplified version of it can be found in Theorem 1 of the next section in this paper. Using the optimal decomposition $K_{i}^{*}, i=1, \ldots, n$, the corresponding super-replicating strategy for the exotic call option has the least price at time zero among a general class of super-replicating investment strategies.

For the moment, we assume that the risk-free rate $r$ is deterministic and constant. In Section 3. we relax this assumption and consider the case where interest rates behave stochastically. From inequality (1) and the discussion above, we find that the optimal super-replicating strategy for an exotic call option consists of buying at time zero $w_{i} \mathrm{e}^{-r\left(T-T_{i}\right)}$ European vanilla call options with pay-off $\left(X_{i}-K_{i}^{*}\right)_{+}$at time $T_{i}$ and holding these options until they expire at time $T_{i}$. We exercise those options with positive pay-offs and invest the eventual pay-offs at that time in the risk-free account until time $T$. The time- 0 price of this optimal super-replicating strategy is given by

$$
\sum_{i=1}^{n} w_{i} \mathrm{e}^{-r\left(T-T_{i}\right)} C_{i}\left[K_{i}^{*}\right]
$$

The upper bound (2) for the time-0 price of an exotic call as well as the corresponding superreplicating strategy can be obtained in an infinite market case, meaning that prices $C_{i}[K]$ of vanilla call options are available for all strikes $K$, and in a finite market case, where only a finite number of vanilla call option prices are observed; see e.g. Hobson et al. (2005) and Chen et al. (2008). In Linders et al. (2012) it is noticed that in the infinite market case, the cheapest superreplicating strategy for the exotic call option derived above cannot be improved by adding other traded derivatives to the financial market, as long as these derivatives are written on a single asset. Remark that in the finite market case this result does not necessarily hold.

The current time- 0 price of a European put option with pay-off $\left(K-X_{i}\right)_{+}$at maturity $T_{i}$ is denoted by $P_{i}[K]$. Assume now that also vanilla put options are traded in the financial market 
and consider the exotic put option with pay-off $(K-S)_{+}$, at time $T$. The inequality

$$
(K-S)_{+}=\left(K-\sum_{i=1}^{n} w_{i} X_{i}\right)_{+} \leq \sum_{i=1}^{n} w_{i}\left(K_{i}-X_{i}\right)_{+}, \quad \mathbb{P} \text {-a.s. }
$$

holds for all $\left(K_{1}, K_{2}, \ldots, K_{n}\right)$ satisfying $\sum_{i=1}^{n} w_{i} K_{i} \geq K$ and $K_{i} \geq 0, i=1, \ldots, n$. Similar

to inequality (1), one can derive an 'optimal' decomposition $K=\sum_{i=1}^{n} w_{i} K_{i}^{*}$ with an explicit expression for the optimal $K_{i}^{*}, i=1, \ldots, n$. Furthermore, we find from (3) that the optimal super-replicating strategy for the exotic put consists of buying a portfolio of European vanilla put options and the time- 0 price is given by

$$
\sum_{i=1}^{n} w_{i} \mathrm{e}^{-r\left(T-T_{i}\right)} P_{i}\left[K_{i}^{*}\right]
$$

For more details we refer to Linders et al. (2012).

Examples of options with a pay-off at time $T$ equal to $(S-K)_{+}$or $(K-S)_{+}$are basket options and Asian options. In the case of a basket option, we have that $T_{i}=T$ and the random variable $X_{i}$ denotes the price level of stock $i$ at time $T$, while $S$ is a weighted sum of the stock price levels at time $T$. In the case of Asian options, only one asset is involved. The random variable $X_{i}$ represents the price level of this asset at time $T-i+1$. The weights $w_{i}$ typically equal $\frac{1}{n}$ such that $S$ is the average price of the asset over the last $n$ periods prior to expiration.

\subsection{The optimization problem}

Hereafter, we always assume that the financial market is arbitrage-free and that there exists a riskneutral pricing measure $\mathbb{Q}$, equivalent to the physical measure $\mathbb{P}$, such that the current price of any pay-off can be represented as the discounted expectation of this pay-off. We further assume for the moment a continuously compounded constant risk-free interest rate. The no-arbitrage 
condition gives rise to the following expressions for the vanilla option prices:

$$
\begin{aligned}
& C_{i}[K]=\mathrm{e}^{-r T_{i}} \mathbb{E}\left[\left(X_{i}-K\right)_{+}\right], \\
& P_{i}[K]=\mathrm{e}^{-r T_{i}} \mathbb{E}\left[\left(K-X_{i}\right)_{+}\right] .
\end{aligned}
$$

In formulae (5) and (6), as well as in the remainder of this section, expectations (distributions) of functions of $\left(X_{1}, \ldots, X_{n}\right)$ have to be understood as expectations (distributions) under the $\mathbb{Q}$ measure. We will often call them risk-neutral expectations (distributions).

We will discuss and further investigate the optimization problem

$$
\min _{K_{1}, \ldots, K_{n}} \sum_{i=1}^{n} w_{i} \mathbb{E}\left[\left(X_{i}-K_{i}\right)_{+}\right] \text {, such that } \sum_{i=1}^{n} w_{i} K_{i}=K
$$

Using expression (5), we see that the solutions $K_{1}^{*}, K_{2}^{*}, \ldots, K_{n}^{*}$ to the minimization problem (7) are related to the cheapest super-replicating strategy for the exotic call option with pay-off $(S-K)_{+}$. Taking into account that

$$
\mathbb{E}\left[\left(K_{i}-X_{i}\right)_{+}\right]=\mathbb{E}\left[\left(X_{i}-K_{i}\right)_{+}\right]+K-\mathbb{E}\left[X_{i}\right], \text { for } K \geq 0
$$

we find that the solutions $K_{1}^{*}, K_{2}^{*}, \ldots, K_{n}^{*}$ to the minimization problem (7) are also solutions to the minimization problem

$$
\min _{K_{1}, \ldots, K_{n}} \sum_{i=1}^{n} w_{i} \mathbb{E}\left[\left(K_{i}-X_{i}\right)_{+}\right], \text {such that } \sum_{i=1}^{n} w_{i} K_{i}=K
$$

From expression (6), we find that the solutions $K_{1}^{*}, K_{2}^{*}, \ldots, K_{n}^{*}$ correspond to the cheapest superreplicating strategy for an exotic put option with pay-off $(K-S)_{+}$, which we considered in (3). From here on, we will solely focus on the optimization problem (7) and, as a result, on the superreplicating strategy for an exotic call option. In Linders et al. (2012), the authors propose an efficient algorithm for determining the upper bounds (2) and (4). They also investigate superreplicating strategies in a unified framework where calls as well as puts are traded. 
The optimization problem (7), which has many applications in finance and insurance, is also discussed in Cheung et al. (2013). Besides the static super-replicating strategies, other applications are optimal capital allocations, see e.g. Dhaene, Tsanakas, Valdez and Vanduffel (2012), and premium calculation from top down, see e.g. Zaks et al. (2006). In Dhaene, Linders, Schoutens and Vyncke (2012), the super-replicating strategies prove to be useful to derive a model-free and forward looking index for the option-implied strength of the co-movement of stock prices.

In this paper, we will investigate three issues. The first issue is the (non-)uniqueness of the optimal solution to (7) and hence to the related static super-replicating strategy. It will be shown that the solution to this problem is not always unique. In the context of capital allocation, the non-uniqueness of the solution to (7), using Lagrange optimization techniques, is investigated in Laeven and Goovaerts (2004).

The second issue that we will investigate is the generalization to a minimization problem with random weights, namely

$$
\min _{K_{1}, \ldots, K_{n}} \sum_{i=1}^{n} w_{i} \mathbb{E}\left[\zeta_{i}\left(X_{i}-K_{i}\right)_{+}\right], \text {such that } \sum_{i=1}^{n} w_{i} K_{i}=K
$$

where the $\zeta_{i}$ are non-negative random variables with $\mathbb{E}\left[\zeta_{i}\right]=1, i=1, \ldots, n$. We further apply these results to the derivation of static super-replicating strategies in a stochastic interest rate setting, in this way generalizing the deterministic interest rate setting of the previous papers.

The third issue that we will investigate is the co-existence of no-arbitrage and comonotonicity of underlying prices. In Chen et al. (2008), the price of the optimal static super-replicating strategy equals the exotic option price when the underlying random variables are comonotonic. An interesting question arises whether or not the comonotonicity property and the no-arbitrage property can co-exist, i.e.does there exist a market situation which is consistent with the observed vanilla option prices and where the price of the exotic option with pay-off $(S-K)_{+}$at maturity $T$ equals the upper bound $\sum_{i=1}^{n} w_{i} \mathrm{e}^{-r\left(T-T_{i}\right)} C_{i}\left[K_{i}^{*}\right]$. It will be shown, for example, that for Asian options, the upper bound is reachable in some cases, but not in general. The problem of the 
multi-asset case, as considered in Dhaene et al.(2013), will also be discussed.

The rest of this paper is organized as follows. In Section 2, the (non-)uniqueness of the optimal solution to the optimization problem (7), and hence of the optimal super-replicating strategy, is discussed. In Section 3, we study the generalized optimization problem (10). As an illustration, we apply the theory to static super-replicating strategies for exotic options in a stochastic interest rate setting. In Section 4, the co-existence of the no-arbitrage assumption and the comonotonicity of the underlying prices is investigated. Section 5 concludes the paper.

\section{2 (Non-)uniqueness of the optimal solution}

\subsection{Basic ideas and the infinite market case}

In this section, we consider the optimization problem (7). The non-uniqueness of the optimal decomposition is proven. Related results can also be found in Cheung et al. (2013). We start with introducing some notations and a basic theorem deriving a particular solution to (7), which can be found e.g. in Dhaene et al. (2002a).

For a given probability level $p \in[0,1]$, we denote the quantile of the random variable $X$ by $F_{X}^{-1}(p)$. As usual, it is defined by

$$
F_{X}^{-1}(p)=\inf \left\{x \in \mathbb{R} \mid F_{X}(x) \geq p\right\}, \quad p \in[0,1]
$$

with $\inf \emptyset=+\infty$ by convention. Hereafter we will also need $\alpha$-mixed inverse distribution function which is introduced in Dhaene et al. (2002a). Therefore, we first define the inverse distribution function $F_{X}^{-1+}(p)$ of a random variable $X$ by

$$
F_{X}^{-1+}(p)=\sup \left\{x \in \mathbb{R} \mid F_{X}(x) \leq p\right\}, \quad p \in[0,1]
$$

with $\sup \emptyset=-\infty$. The $\alpha$-mixed inverse distribution function $F_{X}^{-1(\alpha)}$ of $X$ is defined as the 
following convex combination:

$$
F_{X}^{-1(\alpha)}(p)=\alpha F_{X}^{-1}(p)+(1-\alpha) F_{X}^{-1+}(p), \quad p \in(0,1), \alpha \in[0,1] .
$$

From this definition, one immediately finds that for any random variable $X$ and for all $x$ with $0<F_{X}(x)<1$, there exists an $\alpha_{x} \in[0,1]$ such that

$$
F_{X}^{-1\left(\alpha_{x}\right)}\left(F_{X}(x)\right)=x
$$

A random vector $\underline{X}=\left(X_{1}, \ldots, X_{n}\right)$ is said to be comonotonic if

$$
\underline{X} \stackrel{\mathrm{d}}{=}\left(F_{X_{1}}^{-1}(U), \ldots, F_{X_{n}}^{-1}(U)\right)
$$

where $U$ is a uniform $(0,1)$ r.v. and ' $=$ ' stands for 'equality in distribution'.

For a general random vector $\underline{X}=\left(X_{1}, \ldots, X_{n}\right)$, we call $\left(F_{X_{1}}^{-1}(U), \ldots, F_{X_{n}}^{-1}(U)\right)$ the comonotonic modification of $\underline{X}$, corresponding to the uniform r.v. $U$. Furthermore, for a given set of non-negative weights which are chosen up-front, the weighted sum of the components of the comonotonic modification is denoted by $S^{c}$ :

$$
S^{c}=w_{1} F_{X_{1}}^{-1}(U)+w_{2} F_{X_{2}}^{-1}(U)+\cdots+w_{n} F_{X_{n}}^{-1}(U) .
$$

For an overview of the theory of comonotonicity and its applications in actuarial science and finance, we refer to Dhaene et al. (2002a). Financial and actuarial applications are described in Dhaene et al. (2002b). An updated overview of applications of comonotonicity can be found in Deelstra et al. (2011).

Theorem 1 Assume that $K \in \mathbb{R}$ and consider the minimization problem

$$
\min _{\underline{K}} \sum_{i=1}^{n} w_{i} \mathbb{E}\left[\left(X_{i}-K_{i}\right)_{+}\right], \text {such that } \sum_{i=1}^{n} w_{i} K_{i}=K
$$


where $\underline{K}=\left(K_{1}, \ldots, K_{n}\right)$.

1. If $F_{S^{c}}^{-1+}(0)<K<F_{S^{c}}^{-1}(1)$, a solution $\underline{K}=\left(K_{1}, \ldots, K_{n}\right)$ to the minimization problem (15) is given by

$$
K_{i}=F_{X_{i}}^{-1(\alpha)}\left(F_{S^{c}}(K)\right), \quad i=1, \ldots, n,
$$

while $\alpha \in[0,1]$ follows from

$$
F_{S^{c}}^{-1(\alpha)}\left(F_{S^{c}}(K)\right)=K
$$

2. If $K \leq F_{S^{c}}^{-1+}(0)$, a solution $\underline{K}=\left(K_{1}, \ldots, K_{n}\right)$ to the minimization problem $(15)$ is given by

$$
K_{i}=F_{X_{i}}^{-1+}(0)-e_{i}
$$

with all $e_{i} \geq 0$ and such that $\sum_{i=1}^{n} w_{i} e_{i}=F_{S^{c}}^{-1+}(0)-K$.

3. If $K \geq F_{S^{c}}^{-1}(1)$, a solution $\underline{K}=\left(K_{1}, \ldots, K_{n}\right)$ to the minimization problem 15 is given by

$$
K_{i}=F_{X_{i}}^{-1}(1)+f_{i}
$$

with all $f_{i} \geq 0$ and such that $\sum_{i=1}^{n} w_{i} f_{i}=K-F_{S^{c}}^{-1}(1)$.

The optimization problem (15) and its solution were considered in Dhaene et al. (2002a). Dhaene et al. (2003) study this problem in the particular case that the distribution functions $F_{X_{i}}$, $i=1, \ldots, n$, are strictly increasing. In this case $\alpha=1$ and the solution $(16)$ is obviously unique. A proof of Theorem 1 using Lagrange optimization techniques is given in Laeven and Goovaerts (2004). In the context of pricing Asian options in a Black-Scholes model, Nielsen and Sandman (2003) derived a similar upper bound by means of Lagrange optimization. Hobson et al. (2005) used a Lagrange optimization technique to develop static-arbitrage upper bounds for basket options.

If $F_{S^{c}}^{-1+}(0)<K<F_{S^{c}}^{-1}(1)$, we have that the minimal value of the minimization problem 15 
is given by

$$
\mathbb{E}\left[\left(S^{c}-K\right)_{+}\right]=\sum_{i=1}^{n} w_{i} \mathbb{E}\left[\left(X_{i}-F_{X_{i}}^{-1(\alpha)}\left(F_{S^{c}}(K)\right)\right)_{+}\right],
$$

see e.g. Theorem 7 in Dhaene et al. (2002a). In case $K \leq F_{S^{c}}^{-1+}(0)$, the optimal super-replicating strategy consists of buying for each stock $i$, a vanilla option with strike $K_{i} \leq F_{X_{i}}^{-1+}(0)$. In practice, these options are not traded, but we can replicate its pay-off; see Linders et al. (2012). Furthermore, we have in this particular case that

$$
\left(\sum_{i=1}^{n} w_{i} X_{i}-K\right)_{+}=\sum_{i=1}^{n} w_{i}\left(X_{i}-K_{i}\right)_{+},
$$

which shows that the corresponding strategy replicates the pay-off of the exotic call option. Similarly, we can derive a replicating strategy for the exotic call option with strike $K \geq F_{S^{c}}^{-1}(1)$.

The following example illustrates that the solution given by 16 is not always the unique solution to the minimization problem (15).

Example 1 (Non-uniqueness of the optimal super-replicating strategy) Assume that $n=2$, $K=1, w_{1}=w_{2}=1$ and $\mathbb{Q}\left[X_{i}=0\right]=1-\mathbb{Q}\left[X_{i}=1\right]=\frac{1}{2}$ for $i=1,2$. In this case, we have that $S^{c} \stackrel{\mathrm{d}}{=} 2 X_{1}$. From 20 , we find that the minimum of the constrained minimization problem (15) is given by

$$
\mathbb{E}\left[\left(S^{c}-1\right)_{+}\right]=\frac{1}{2}
$$

As $F_{S^{c}}(1)=\frac{1}{2}$, we find from 17 that

$$
\alpha=\frac{1}{2}
$$

This leads to the conclusion that the optimal solution $(16)$ is given by

$$
K_{i}=\frac{1}{2}, \quad i=1,2
$$

whereas the constrained minimum of the objective function is given by $\frac{1}{2}$. 
Now, for any couple $\left(K_{1}, 1-K_{1}\right)$ with $K_{1} \in(0,1)$, we find

$$
\mathbb{E}\left[\left(X_{1}-K_{1}\right)_{+}\right]+\mathbb{E}\left[\left(X_{2}-\left(1-K_{1}\right)\right)_{+}\right]=\frac{1}{2}\left(1-K_{1}\right)+\frac{1}{2}\left(1-\left(1-K_{1}\right)\right)=\frac{1}{2}
$$

We can conclude that any couple $\left(K_{1}, 1-K_{1}\right)$ with $K_{1} \in(0,1)$ is a solution to the optimization problem and hence, the solution given by $(16)$ is not always the unique solution to the minimization problem (15).

The set of all solutions to the constrained optimization problem $(15)$ is derived in the next theorem.

Theorem 2 For $K \in \mathbb{R}$, the set of all solutions $\underline{K}=\left(K_{1}, \ldots, K_{n}\right)$ to the minimization problem (15) is given by

$$
A=\left\{\underline{K} \mid \sum_{i=1}^{n} w_{i} K_{i}=K \text { and } F_{X_{i}}\left(K_{i}\right)=F_{S^{c}}(K) ; i=1,2, \ldots, n\right\}
$$

Proof. We will give the proof for the bivariate case. A generalization to the $n$-dimensional case is straightforward. For $i=1,2$, we introduce the following notation:

$$
K_{i}^{*}=\left\{\begin{array}{cc}
F_{X_{i}}^{-1+}(0)-e_{i}, & \text { if } K \leq F_{S^{c}}^{-1+}(0) \\
F_{X_{i}}^{-1(\alpha)}\left(F_{S^{c}}(K)\right), & \text { if } K \in\left(F_{S^{c}}^{-1+}(0), F_{S^{c}}^{-1}(1)\right) \\
F_{X_{i}}^{-1}(1)+f_{i}, & \text { if } K \geq F_{S^{c}}^{-1}(1)
\end{array}\right.
$$

where the non-negative constants $e_{i}$ and $f_{i}$ are defined respectively as in (18) and (19), and $\alpha$ is chosen as in (17).

It follows from Theorem 1 that $\left(K_{1}^{*}, K_{2}^{*}\right)$ is a solution of the minimization problem 15 . Furthermore, we have that $\left(K_{1}^{*}, K_{2}^{*}\right) \in A$. Notice that for any $K$, the stop-loss premium $\mathbb{E}\left[\left(X_{i}-K\right)_{+}\right]$ can be expressed as

$$
\mathbb{E}\left[\left(X_{i}-K\right)_{+}\right]=\int_{K}^{+\infty}\left(1-F_{X_{i}}(x)\right) \mathrm{d} x .
$$


(a) We will first prove that in the case $\underline{K}=\left(K_{1}, K_{2}\right) \in A$, we have that $\underline{K}$ is a solution to the minimization problem (15).

- In the case $K_{1}=K_{1}^{*}$, we find from $w_{1} K_{1}+w_{2} K_{2}=K$ that also $K_{2}=K_{2}^{*}$, so that $\left(K_{1}, K_{2}\right)$ is indeed a solution to the minimization problem.

- Let us now consider the case where $K_{1}<K_{1}^{*}$, which is illustrated graphically in Figure 1. Because $w_{1} K_{1}+w_{2} K_{2}=w_{1} K_{1}^{*}+w_{2} K_{2}^{*}$, we must have that $K_{2}>K_{2}^{*}$. Using expression 22 for $\mathbb{E}\left[\left(X_{1}-K_{1}\right)_{+}\right]$as well as for $\mathbb{E}\left[\left(X_{1}-K_{1}^{*}\right)_{+}\right]$, and noting that $F_{X_{1}}(x)=F_{S^{c}}(K)$ for all $x \in\left[K_{1}, K_{1}^{*}\right]$, we find that

$$
\mathbb{E}\left[\left(X_{1}-K_{1}\right)_{+}\right]=\left(K_{1}^{*}-K_{1}\right)\left(1-F_{S^{c}}(K)\right)+\mathbb{E}\left[\left(X_{1}-K_{1}^{*}\right)_{+}\right]
$$

Similarly, from $F_{X_{2}}(x)=F_{S^{c}}(K)$ for all $x \in\left[K_{2}^{*}, K_{2}\right]$, we find

$$
\mathbb{E}\left[\left(X_{2}-K_{2}\right)_{+}\right]=\mathbb{E}\left[\left(X_{2}-K_{2}^{*}\right)_{+}\right]-\left(K_{2}-K_{2}^{*}\right)\left(1-F_{S^{c}}(K)\right)
$$

The requirement $w_{1} K_{1}+w_{2} K_{2}=K=w_{1} K_{1}^{*}+w_{2} K_{2}^{*}$ implies that $w_{1}\left(K_{1}^{*}-K_{1}\right)=$ $w_{2}\left(K_{2}-K_{2}^{*}\right)$. Hence,

$w_{1} \mathbb{E}\left[\left(X_{1}-K_{1}\right)_{+}\right]+w_{2} \mathbb{E}\left[\left(X_{2}-K_{2}\right)_{+}\right]=w_{1} \mathbb{E}\left[\left(X_{1}-K_{1}^{*}\right)_{+}\right]+w_{2} \mathbb{E}\left[\left(X_{2}-K_{2}^{*}\right)_{+}\right]$.

We can conclude that $\underline{K}=\left(K_{1}, K_{2}\right) \in A$ implies that $\underline{K}$ is a solution to the minimization problem (15).

- In a similar way, one can prove that if $K_{1}>K_{1}^{*}$, it holds that $\left(K_{1}, K_{2}\right) \in A$ implies that $\underline{K}$ is a solution to the minimization problem $(15)$.

(b) Next, we will prove that if $\underline{K}=\left(K_{1}, K_{2}\right) \notin A$, then $\underline{K}$ cannot be a solution to the minimization problem (15).

- In the case $w_{1} K_{1}+w_{2} K_{2} \neq K$, clearly $\underline{K}$ cannot be a solution to the minimization 
problem (15).

- For $K \in\left(F_{S^{c}}^{-1+}(0), F_{S^{c}}^{-1}(1)\right)$, it holds that $F_{S^{c}}(K) \in(0,1)$. Assume that $F_{X_{1}}\left(K_{1}\right)<$ $F_{S^{c}}(K)$. This case is illustrated graphically in Figure 2 .

We immediately find that $K_{1}<K_{1}^{*}$. Taking into account that $F_{X_{1}}\left(K_{1}\right)<F_{S^{c}}(K)$, we arrive at

$$
\mathbb{E}\left[\left(X_{1}-K_{1}\right)_{+}\right]>\left(K_{1}^{*}-K_{1}\right)\left(1-F_{S^{c}}(K)\right)+\mathbb{E}\left[\left(X_{1}-K_{1}^{*}\right)_{+}\right]
$$

Because $w_{1} K_{1}+w_{2} K_{2}=K=w_{1} K_{1}^{*}+w_{2} K_{2}^{*}$, it should hold that $K_{2}>K_{2}^{*}$. This leads to $F_{X_{2}}\left(K_{2}\right) \geq F_{S^{c}}(K)$. Hence, we find

$$
\mathbb{E}\left[\left(X_{2}-K_{2}\right)_{+}\right] \geq \mathbb{E}\left[\left(X_{2}-K_{2}^{*}\right)_{+}\right]-\left(K_{2}-K_{2}^{*}\right)\left(1-F_{S^{c}}(K)\right)
$$

From these expressions and $w_{1}\left(K_{1}^{*}-K_{1}\right)=w_{2}\left(K_{2}-K_{2}^{*}\right)$, we conclude that

$w_{1} \mathbb{E}\left[\left(X_{1}-K_{1}\right)_{+}\right]+w_{2} \mathbb{E}\left[\left(X_{2}-K_{2}\right)_{+}\right]>w_{1} \mathbb{E}\left[\left(X_{1}-K_{1}^{*}\right)_{+}\right]+w_{2} \mathbb{E}\left[\left(X_{2}-K_{2}^{*}\right)_{+}\right]$,

implying that $\underline{K}$ with $w_{1} K_{1}+w_{2} K_{2}=K$ and $F_{X_{1}}\left(K_{1}\right)<F_{S^{c}}(K)$ cannot be a solution to the minimization problem (15).

- If $K \in\left(F_{S^{c}}^{-1+}(0), F_{S^{c}}^{-1}(1)\right)$, the cases $\left(w_{1} K_{1}+w_{2} K_{2}=K\right.$ and $F_{X_{1}}\left(K_{1}\right)>$ $\left.F_{S^{c}}(K)\right),\left(w_{1} K_{1}+w_{2} K_{2}=K\right.$ and $\left.F_{X_{2}}\left(K_{2}\right)<F_{S^{c}}(K)\right),\left(w_{1} K_{1}+w_{2} K_{2}=K\right.$ and $\left.F_{X_{2}}\left(K_{2}\right)<F_{S^{c}}(K)\right)$ and $\left(w_{1} K_{1}+w_{2} K_{2}=K\right.$ and $\left.F_{X_{2}}\left(K_{2}\right)>F_{S^{c}}(K)\right)$ can be proven in a similar way.

- Assume now that $K \leq F_{S^{c}}^{-1+}(0)$. This implies that $F_{S^{c}}(K)=0$. If $w_{1} K_{1}+w_{2} K_{2}=$ $K$, then $\left(K_{1}, K_{2}\right) \notin A$ can only hold if either $F_{X_{1}}\left(K_{1}\right)>0$ or $F_{X_{2}}\left(K_{2}\right)>0$. Assume for the moment that $F_{X_{1}}\left(K_{1}\right)>0$, so $K_{1}>F_{X_{1}}^{-1+}(0)$. The equalities $w_{1} K_{1}+$ $w_{2} K_{2}=K$ and $w_{1} F_{X_{1}}^{-1+}(0)+w_{2} F_{X_{2}}^{-1+}(0)=F_{S^{c}}^{-1+}(0)$ imply that $K_{2}<F_{X_{2}}^{-1+}(0)$. 


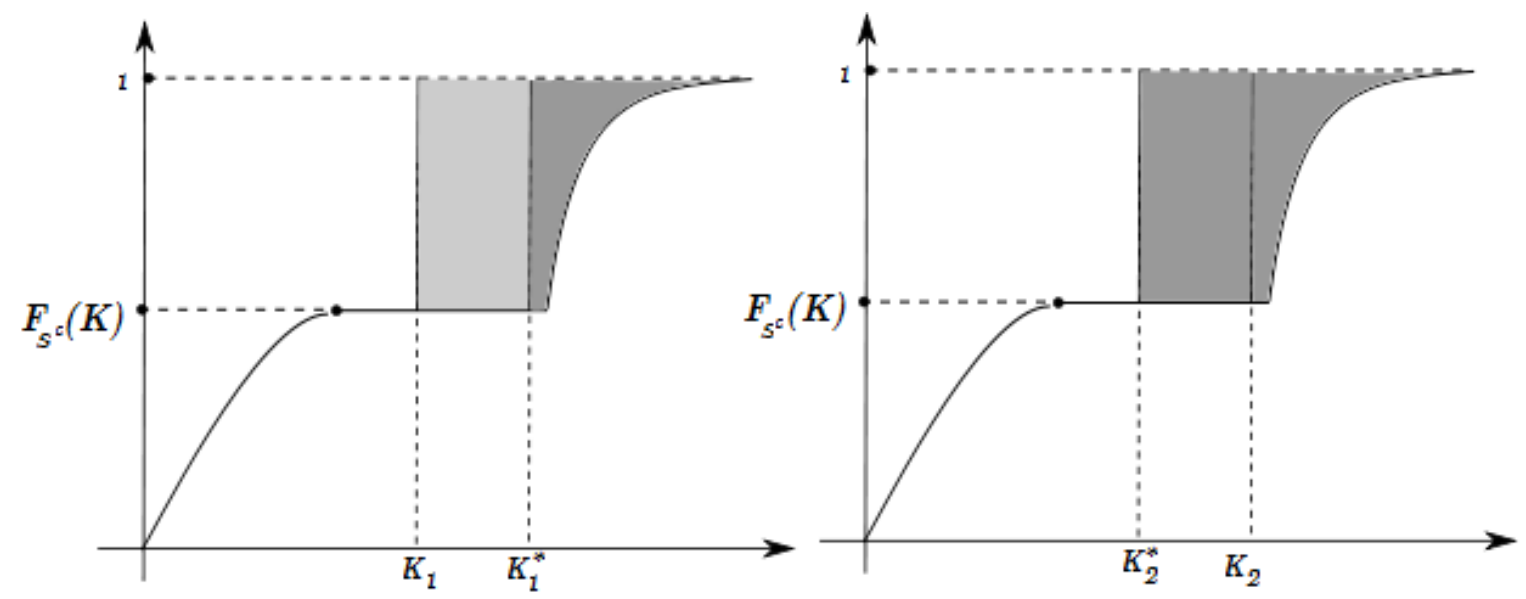

Figure 1: $F_{X_{1}}$ (on the left), $F_{X_{2}}$ on the right), $\underline{K} \in A$ and $K_{1}<K_{1}^{*}$.

Then we find that

$$
\begin{aligned}
w_{1} \mathbb{E}\left[\left(X_{1}-K_{1}\right)_{+}\right]+w_{2} \mathbb{E}\left[\left(X_{2}-K_{2}\right)_{+}\right] & =w_{1} \mathbb{E}\left[\left(X_{1}-K_{1}\right)_{+}\right]+w_{2} \mathbb{E}\left[X_{2}-K_{2}\right] \\
& >w_{1} \mathbb{E}\left[X_{1}-K_{1}\right]+w_{2} \mathbb{E}\left[X_{2}-K_{2}\right] \\
& =\mathbb{E}\left[w_{1} X_{1}+w_{2} X_{2}\right]-K \\
& =w_{1} \mathbb{E}\left[\left(X_{1}-K_{1}^{*}\right)_{+}\right]+w_{2} \mathbb{E}\left[\left(X_{2}-K_{2}^{*}\right)_{+}\right],
\end{aligned}
$$

which proves that $\left(K_{1}, K_{2}\right)$ cannot be an optimal solution.

- The situations where $\left(K \leq F_{S^{c}}^{-1+}(0), w_{1} K_{1}+w_{2} K_{2}=K, F_{X_{2}}\left(K_{2}\right)>0\right),(K \geq$ $\left.F_{S^{c}}^{-1}(1), w_{1} K_{1}+w_{2} K_{2}=K, F_{X_{1}}\left(K_{1}\right)<1\right)$ and $\left(K \geq F_{S^{c}}^{-1}(1), w_{1} K_{1}+w_{2} K_{2}=K\right.$, $\left.F_{X_{2}}\left(K_{2}\right)<1\right)$ can be proven in a similar way.

In the remainder of this paper, we always silently assume that $F_{S^{c}}^{-1+}(0)<K<F_{S^{c}}^{-1}(1)$, unless stated otherwise. In this case, the set $A$ in Theorem 1 can also be expressed as

$$
A=\left\{\underline{K} \mid \sum_{i=1}^{n} w_{i} K_{i}=K \text { and } K_{i}=F_{X_{i}}^{-1\left(\alpha_{i}\right)}\left(F_{S^{c}}(K)\right) \text { for some } \alpha_{i} \in[0,1] ; i=1,2, \ldots, n\right\} .
$$




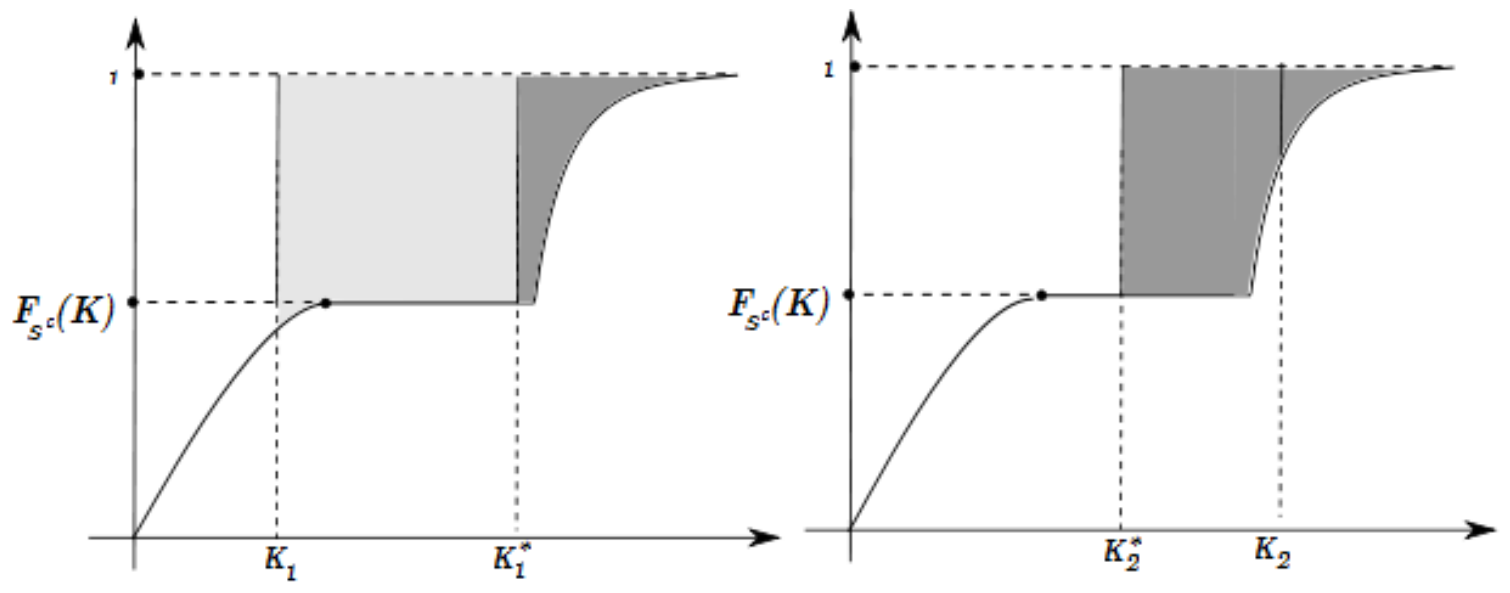

Figure 2: $F_{X_{1}}$ (on the left), $F_{X_{2}}$ (on the right), $\underline{K} \notin A, w_{1} K_{1}+w_{2} K_{2}=K$ and $F_{X_{1}}\left(K_{1}\right)<$ $F_{S^{c}}(K)$.

From the expression above we can conclude that the set $A$ reduces to the singleton $A=$ $\left\{\left(F_{X_{1}}^{-1}\left(F_{S^{c}}(K)\right), F_{X_{2}}^{-1}\left(F_{S^{c}}(K)\right), \ldots, F_{X_{n}}^{-1}\left(F_{S^{c}}(K)\right)\right)\right\}$ in case all marginal distributions $F_{X_{i}}$ are strictly increasing.

Notice that the solution to the minimization problem $[15]$ is unique in the set $B$ defined by

$$
B=\left\{\underline{K} \mid \sum_{i=1}^{n} w_{i} K_{i}=K \text { and } K_{i}=F_{X_{i}}^{-1(\alpha)}\left(F_{S^{c}}(K)\right) ; i=1,2, \ldots, n \text {, for some } \alpha \in[0,1]\right\}
$$

We can conclude that the minimization problem (7) does not always have a unique solution. In the super-replicating context, this means that there is not always a unique optimal superreplicating strategy in the infinite market case (where European call option prices for all possible strikes are available), except when all risk-neutral marginal distributions $F_{X_{i}}$ are strictly increasing.

\subsection{The finite market case}

In practice, only a finite number of strikes are traded for each underlying. Therefore, we assume that for asset $i, i=1,2, \ldots, n$, at current time 0 , European call options with strikes $0=K_{i, 0}<$ $K_{i, 1}<\ldots<K_{i, m_{i}}<F_{X_{i}}^{-1}(1)$ and maturity $T_{i}$ are available in the market. The prices of these 
options are denoted by $C_{i}\left[K_{i, j}\right], i=1,2, \ldots, n ; j=0,1, \ldots, m_{i}$. Furthermore, we assume that $F_{X_{i}}^{-1}(1)$ is known and finite. We will denote this 'maximal value' of $X_{i}$ by $K_{i, m_{i}+1}$.

When option prices $C_{i}[K]$ are available for any strike $K$, we can derive the implied riskneutral distribution $F_{X_{i}}$ of the price of underlying $i$ at time $T_{i}$ as follows

$$
F_{X_{i}}(x)=1+\mathrm{e}^{r T_{i}} C_{i}^{\prime}[x+]
$$

where $C_{i}^{\prime}[x+]$ denotes the right derivative of $C_{i}$ in $x$.

Because we assumed that there are only a finite number of traded strikes for underlying $i$, the call option curve $C_{i}$ is not fully specified and therefore $F_{X_{i}}$ is not completely specified. We circumvent this problem by approximating the partially known convex call option curve $C_{i}$ by the piecewise linear convex function $\bar{C}_{i}$ connecting the observed points $\left(K_{i, j}, C_{i}\left[K_{i, j}\right]\right), j=$ $0,1, \ldots, m_{i}+1$. Obviously, any $\bar{C}_{i}[K]$ is an upper bound for the corresponding call option price $C_{i}[K]$ and both values are identical if $K$ is a traded strike. We can then find the cdf $\bar{F}_{X_{i}}$ which corresponds to the option curve $\bar{C}_{i}$ from the following relation:

$$
\bar{F}_{X_{i}}(x)=1+\mathrm{e}^{r T_{i}} \bar{C}_{i}^{\prime}[x+]
$$

The optimal super-replicating strategy for the exotic call option with pay-off $(S-K)_{+}$at time $T$ follows from the minimization problem $(15)$. A possible solution is given in Theorem 1. Taking into account that only partial option data are available, it is not possible to solve the minimization problem (15). However, we can solve the following minimization problem:

$$
\min _{\underline{K}} \sum_{i=1}^{n} w_{i} \mathbb{E}\left[\left(\bar{F}_{X_{i}}^{-1}(U)-K_{i}\right)_{+}\right] \text {, such that } \sum_{i=1}^{n} w_{i} K_{i}=K
$$

Let $\bar{S}^{c}$ denote the comonotonic sum $\sum_{i=1}^{n} w_{i} \bar{F}_{X_{i}}^{-1}(U)$, then 27 can be solved using Theorem 1. The above-mentioned procedure for coping with the finite market case was firstly proposed in Hobson et al. (2005). Simplified proofs for their results were presented in Chen et al. (2008). A 


\begin{tabular}{|c|c|c|c|}
\hline$K$ & $C_{A}[K]$ & K & $C_{B}[K]$ \\
\hline 0 & 45.5 & 0 & 35.5 \\
\hline 40 & 9.8 & 35 & 5.02 \\
\hline 45 & 5.5 & 37.5 & 3.05 \\
\hline 50 & 2.09 & 40 & 1.83 \\
\hline 55 & 0.37 & 42.5 & 0.97 \\
\hline 60 & 0.08 & 45 & 0.29 \\
\hline 65 & 0.03 & 47.5 & 0.08 \\
\hline
\end{tabular}

Table 1: Observed vanilla call option prices for asset A (left table) and observed vanilla call option prices for asset $\mathrm{B}$ (right table).

more general set-up as well as a detailed algorithm for determining the solution to the minimization problem (27) numerically, is given in Linders et al. (2012). Let us show the non-uniqueness of the super-replicating strategy in the finite market case through an example.

Example 2 Suppose the European call option prices for asset A and asset B, as listed in Table 1 . can be observed in the market at time zero. Further, suppose that there is a basket option written on a combination of asset $\mathrm{A}$ and $\mathrm{B}$, with weight factors $w_{1}=w_{2}=1 / 2$, strike $K=47.5$ and maturity $T=1$. The continuously compound yearly interest rate $r$ follows from $\mathrm{e}^{r T}=1.04$. The pay-off function of the basket call option is given by

$$
\left(\frac{1}{2} X_{1}+\frac{1}{2} X_{2}-47.5\right)_{+}
$$

From expression (26), we find that for any $i=1,2$ and $j=0,1, \ldots, 6$, we have

$$
\bar{F}_{X_{i}}\left(K_{i, j}\right)=1+\mathrm{e}^{r T} \frac{C_{i}\left[K_{i, j+1}\right]-C_{i}\left[K_{i, j}\right]}{K_{i, j+1}-K_{i, j}} .
$$

For any $p \in(0,1)$, we have that $\bar{F}_{X_{i}}^{-1}(p)$ is given by

$$
\bar{F}_{X_{i}}^{-1}(p)=K_{i, j} \text { if } \bar{F}_{X_{i}}\left(K_{i, j-1}\right)<p \leq \bar{F}_{X_{i}}\left(K_{i, j}\right), \quad j=0,1, \ldots, 6
$$


Let $\bar{S}^{c}$ be equal to $w_{1} \bar{F}_{X_{1}}^{-1}(U)+w_{2} \bar{F}_{X_{2}}^{-1}(U)$. As the couple $(50,40)$ is an element of the support of $\left(\bar{F}_{X_{1}}^{-1}(U), \bar{F}_{X_{2}}^{-1}(U)\right)$, we find that

$$
F_{\bar{S}^{c}}(45)=\mathbb{Q}\left[\bar{F}_{X_{1}}^{-1}(U) \leq 50, \bar{F}_{X_{2}}^{-1}(U) \leq 40\right]=0.6422
$$

see Lemma 5 in Linders et al. (2012). Furthermore, we can verify that

$$
\begin{aligned}
& \bar{F}_{X_{1}}^{-1}\left(F_{\bar{S}^{c}}(45)\right)=50 \text { and } \bar{F}_{X_{1}}^{-1+}\left(F_{\bar{S}^{c}}(45)\right)=55, \\
& \bar{F}_{X_{2}}^{-1}\left(F_{\bar{S}^{c}}(45)\right)=40 \text { and } \bar{F}_{X_{2}}^{-1+}\left(F_{\bar{S}^{c}}(45)\right)=42.5 .
\end{aligned}
$$

Using these equalities, we can check that there exists a value $\alpha \in(0,1)$ such that

$$
w_{1} \bar{F}_{X_{1}}^{-1(\alpha)}\left(F_{\bar{S}^{c}}(45)\right)+w_{2} \bar{F}_{X_{2}}^{-1(\alpha)}\left(F_{\bar{S}^{c}}(45)\right)=47.5
$$

We find that $\alpha=1 / 3$ and $F_{\bar{S}^{c}}(K)=F_{\bar{S}^{c}}(45)$. The optimal strike prices are $K_{1}=53.3333$ and $K_{2}=41.6667$. The precision for $K_{1}$ and $K_{2}$ is to the 4 th decimal point. Since we are considering the finite market case, the price of the optimal strategy at time zero is

$$
\begin{aligned}
w_{1} \bar{C}_{1}\left[K_{1}^{*}\right]+w_{2} \bar{C}_{2}\left[K_{2}^{*}\right]= & w_{1}\left(\alpha C_{1}\left[\bar{F}_{X_{1}}^{-1}\left(F_{\bar{S}^{c}}(K)\right)\right]+(1-\alpha) C_{1}\left[\bar{F}_{X_{1}}^{-1+}\left(F_{\bar{S}^{c}}(K)\right)\right]\right) \\
& +w_{2}\left(\alpha C_{2}\left[\bar{F}_{X_{2}}^{-1}\left(F_{\bar{S}^{c}}(K)\right)\right]+(1-\alpha) C_{2}\left[\bar{F}_{X_{2}}^{-1+}\left(F_{\bar{S}^{c}}(K)\right)\right]\right) \\
= & \frac{1}{2}\left(\frac{1}{3} \cdot 2.09+\frac{2}{3} \cdot 0.37\right)+\frac{1}{2}\left(\frac{1}{3} \cdot 1.83+\frac{2}{3} \cdot 0.97\right) \\
= & 1.1 .
\end{aligned}
$$

Given $F_{\bar{S}^{c}}(K)=0.6422$, we can also have that $F_{X_{1}}^{-1\left(\alpha_{1}\right)}\left(F_{\bar{S}^{c}}(K)\right) \in[52.5,55]$, when $\alpha_{1} \in\left[0, \frac{1}{2}\right]$ and $F_{X_{2}}^{-1\left(\alpha_{2}\right)}\left(F_{\bar{S}^{c}}(K)\right)=95-F_{X_{1}}^{-1\left(\alpha_{1}\right)}\left(F_{\bar{S}^{c}}(K)\right)$, when $\alpha_{2}=1-2 \alpha_{1}$. Hence, from 23 and chosing $\alpha_{1}=\frac{1}{6}$ and $\alpha_{2}=\frac{2}{3}$, it follows that $\left(K_{1}, K_{2}\right)=(54.1667,40.8333)$ is a solution to the constrained minimization problem (27) as well. The price of this optimal choice at time zero 
equals 1.1. Indeed,

$$
\frac{1}{2}\left(\frac{1}{6} \cdot 2.09+\frac{5}{6} \cdot 0.37\right)+\frac{1}{2}\left(\frac{2}{3} \cdot 1.83+\frac{1}{3} \cdot 0.97\right)=1.1
$$

In the next section, we consider a generalization of the optimization problem $(15)$ which can be applied to determine super-replicating strategies in a stochastic interest rate setting.

\section{A generalized constrained minimization problem}

In Hobson et al. (2005) and Chen et al. (2008), the discussion above was carried out in a deterministic interest rate setting. The generalization to the stochastic interest rate world requires the study of the optimization problem $(10)$ under the $\mathbb{Q}$-measure, which we repeat here:

$$
\min _{K_{1}, \ldots, K_{n}} \sum_{i=1}^{n} w_{i} \mathbb{E}\left[\zeta_{i}\left(X_{i}-K_{i}\right)_{+}\right], \text {such that } \sum_{i=1}^{n} w_{i} K_{i}=K
$$

where the $\zeta_{i}$ are non-negative random variables with $\mathbb{E}\left[\zeta_{i}\right]=1, i=1, \ldots, n$. When all $\zeta_{i}$ are identical to 1 , the problem reduces to the one in the previous section.

\subsection{Derivation of the optimal solution}

The optimization problem (28) is considered in Dhaene, Tsanakas, Valdez and Vanduffel (2012) where an optimal allocation problem is studied and a particular set of optimal allocations $K_{i}$, $i=1, \ldots, n$, is derived. Here, we restate the results of that paper, give an alternative proof of the claim in Lemma 3 and characterize the complete solution set in (38).

The solution to the general optimization problem 28 is expressed in terms of functions $F_{X_{i}}^{\left(\zeta_{i}\right)}$, which are defined as follows:

$$
F_{X_{i}}^{\left(\zeta_{i}\right)}(x)=\mathbb{E}\left[\zeta_{i} \mathbb{I}\left\{X_{i} \leq x\right\}\right]=\mathbb{E}\left[\zeta_{i} \mid X_{i} \leq x\right] F_{X_{i}}(x), \quad i=1, \ldots, n
$$


Each function $F_{X_{i}}^{\left(\zeta_{i}\right)}$ defines a proper distribution function and we call this distribution function the $\zeta_{i}$-weighted distribution of $X_{i}$. More information can be found in Rao (1997), Furman and Zitikis (2008) and the references therein. The corresponding decumulative distribution function is given by

$$
1-F_{X_{i}}^{\left(\zeta_{i}\right)}(x)=\mathbb{E}\left[\zeta_{i} \mathbb{I}\left\{X_{i}>x\right\}\right]=\mathbb{E}\left[\zeta_{i} \mid X_{i}>x\right]\left(1-F_{X_{i}}(x)\right), \quad i=1, \ldots, n
$$

A sufficient condition for $F_{X_{i}}^{\left(\zeta_{i}\right)}$ to be continuous is that $F_{X_{i}}$ is continuous. A sufficient condition for $F_{X_{i}}^{\left(\zeta_{i}\right)}$ to be strictly increasing is that $F_{X_{i}}$ is strictly increasing and $\mathbb{Q}\left[\zeta_{i}>0\right]=1$.

The following lemma will play an important role to derive the set of solutions to (28). Here we give an alternative proof based on a change of measures, a method which is well-known in finance and also used in a dynamic setting when the Radon-Nikodym derivative is strictly positive; see e.g. Geman et al. (1995).

Lemma 3 Let $U$ be a random variable which is uniformly distributed on the unit interval $(0,1)$ of some probability space, then it holds that

$$
\mathbb{E}\left[\zeta_{i}\left(X_{i}-K_{i}\right)_{+}\right]=\mathbb{E}^{\operatorname{Pr}}\left[\left(\left(F_{X_{i}}^{\left(\zeta_{i}\right)}\right)^{-1}(U)-K_{i}\right)_{+}\right], \quad i=1, \ldots, n,
$$

where the super index $\operatorname{Pr}$ is the probability measure in that probability space.

Proof. This lemma follows by the theorem of Radon-Nikodym. $\mathbb{E}[\cdot]$ is calculated based on the probability space $(\Omega, \mathcal{F}, \mathbb{Q})$. One can interpret $\zeta_{i}$ as a Radon-Nikodym derivative since $\mathbb{Q}\left[\zeta_{i}>0\right]=1$ and $\mathbb{E}\left[\zeta_{i}\right]=1$. Let us denote $\mathbb{Q}_{i}$ such that

$$
\mathbb{Q}_{i}[A]=\mathbb{E}\left[\zeta_{i} \mathbb{I}_{A}\right] \quad i=1, \ldots, n
$$

for all $A \in \mathcal{F}$. Then,

$$
\mathbb{E}\left[\zeta_{i}\left(X_{i}-K_{i}\right)_{+}\right]=\mathbb{E}_{\mathbb{Q}_{i}}\left[\left(X_{i}-K_{i}\right)_{+}\right], \quad i=1, \ldots, n
$$


where we now need to find the law of $X_{i}$ under $\mathbb{Q}_{i}$.

Denoting $X_{i} \sim F_{X_{i}}$ under $\mathbb{Q}$, according to (29) and (32), we find that

$$
\mathbb{Q}_{i}\left[X_{i} \leq x\right]=\mathbb{E}\left[\zeta_{i} \mathbb{I}\left\{X_{i} \leq x\right\}\right]=F_{X_{i}}^{\left(\zeta_{i}\right)}(x), \quad i=1, \ldots, n
$$

Since with $U$ a uniformly distributed random variable under $\operatorname{Pr}$, we have that

$$
F_{X_{i}}^{\left(\zeta_{i}\right)}(x)=\operatorname{Pr}\left[U \leq F_{X_{i}}^{\left(\zeta_{i}\right)}(x)\right]=\operatorname{Pr}\left[\left(F_{X_{i}}^{\left(\zeta_{i}\right)}\right)^{-1}(U) \leq x\right], \quad i=1, \ldots, n
$$

from which we conclude that $X_{i}$ has the same law under $\mathbb{Q}_{i}$ as $\left(F_{X_{i}}^{\left(\zeta_{i}\right)}\right)^{-1}(U)$ under $\operatorname{Pr}$. Therefore, it holds that

$$
\mathbb{E}_{\mathbb{Q}_{i}}\left[\left(X_{i}-K_{i}\right)_{+}\right]=\mathbb{E}^{P r}\left[\left(\left(F_{X_{i}}^{\left(\zeta_{i}\right)}\right)^{-1}(U)-K_{i}\right)_{+}\right], \quad i=1, \ldots, n
$$

Combining this result with 33 we arrive at the stated result.

Note that when $U$ exists on the probability space $(\Omega, \mathcal{F}, \mathbb{Q}), \operatorname{Pr}$ equals $\mathbb{Q}$ and the superscript can be omitted in Equation (31). We should emphasize that it is possible that $U$ does not exist on $(\Omega, \mathcal{F}, \mathbb{Q})$, see Dhaene and Kukush (2011).

For notational simplicity, we assume that $U$ exists on $(\Omega, \mathcal{F}, \mathbb{Q})$ from here on.

A solution to the generalized optimization problem $(28)$ is derived in the following theorem.

Theorem 4 Let $\bar{S}^{c}$ be the comonotonic sum defined by

$$
\bar{S}^{c}=\sum_{i=1}^{n} w_{i}\left(F_{X_{i}}^{\left(\zeta_{i}\right)}\right)^{-1}(U)
$$

where the random variable $U$ is uniformly distributed on the unit interval $(0,1)$. In the case $F_{\bar{S}^{c}}^{-1+}(0)<K<F_{\bar{S}^{c}}^{-1}(1)$, the optimization problem $(28)$ has the following solution:

$$
K_{i}=\left(F_{X_{i}}^{\left(\zeta_{i}\right)}\right)^{-1(\alpha)}\left(F_{\bar{S}^{c}}(K)\right), \quad i=1, \ldots, n
$$


where $\alpha \in[0,1]$ follows from

$$
F_{\bar{S}^{c}}^{-1(\alpha)}\left(F_{\bar{S}^{c}}(K)\right)=K
$$

Proof. From Lemma 3, we find that the optimization problem (28) can be rewritten as

$$
\min _{K_{1}, \ldots, K_{n}} \sum_{i=1}^{n} w_{i} \mathbb{E}\left[\left(\left(F_{X_{i}}^{\left(\zeta_{i}\right)}\right)^{-1}(U)-K_{i}\right)_{+}\right] \text {, such that } \sum_{i=1}^{n} w_{i} K_{i}=K
$$

The stated result follows then by applying Theorem 1 .

Note that it is straightforward to extend Theorem 4 to include the case where

$K \notin\left(F_{\bar{S}^{c}}^{-1+}(0), F_{\bar{S}^{c}}^{-1}(1)\right)$. It should also be noted that the non-uniqueness of the optimal solution holds in this general setting as well. According to Theorem 2 , the set of all solutions $\underline{K}=$ $\left(K_{1}, \ldots, K_{n}\right)$ to the minimization problem 28$)$ is given by

$$
A=\left\{\underline{K} \mid \sum_{i=1}^{n} w_{i} K_{i}=K \text { and } F_{X_{i}}^{\left(\zeta_{i}\right)}\left(K_{i}\right)=F_{\bar{S}^{c}}(K) ; i=1,2, \ldots, n\right\}
$$

or, equivalently,

$$
A=\left\{\begin{array}{c}
\underline{K} \mid \sum_{i=1}^{n} w_{i} K_{i}=K \text { and } K_{i}=\left(F_{X_{i}}^{\left(\zeta_{i}\right)}\right)^{-1\left(\alpha_{i}\right)}\left(F_{\bar{S}^{c}}(K)\right) \\
\text { for some } \alpha_{i} \in[0,1] ; i=1,2, \ldots, n
\end{array}\right\}
$$

If $\mathbb{Q}\left[\zeta_{i}>0\right]=1, i=1, \ldots, n$, and the distributions $F_{X_{i}}^{\left(\zeta_{i}\right)}$ are strictly increasing, the optimal allocations 37 reduce to

$$
K_{i}=\left(F_{X_{i}}^{\left(\zeta_{i}\right)}\right)^{-1}\left(F_{\bar{S}^{c}}(K)\right), \quad i=1, \ldots, n
$$

\subsection{Application to a stochastic interest rate setting}

In this section we generalize the problem of finding static super-replicating strategies for a class of exotic options from the deterministic interest rate setting to the stochastic interest rate world. 
We recall that expectations are taken with respect to the pricing measure $\mathbb{Q}$, unless explicitly stated otherwise.

\subsubsection{Interest rate process and zero-coupon bond}

Consider an adapted interest rate process $\{R(t) \mid t \geq 0\}$ defined on a filtered probability space $\left(\Omega, \mathcal{F},\left\{\mathcal{F}_{t}\right\}_{0 \leq t \leq T}, \mathbb{P}\right)$. The corresponding discount process $\{D(t) \mid t \geq 0\}$ is given by

$$
D(t)=\mathrm{e}^{-\int_{0}^{t} R(u) \mathrm{d} u}
$$

Obviously $D(0)=1$. Assume that there are no arbitrage opportunities and that the market prices of all derivatives involved are given by expectations of discounted pay-offs under the pricing measure $\mathbb{Q}$. Further, consider a zero-coupon bond that pays 1 unit of currency at maturity $T$. The value of this bond at time $t \in[0, T]$ is denoted by $P(t, T)$ and can be expressed as

$$
P(t, T)=\frac{1}{D(t)} \mathbb{E}\left[D(T) \mid \mathcal{F}_{t}\right]
$$

In particular $P(T, T)=1$ and $P(0, T)=\mathbb{E}[D(T)]$. The current time zero prices of the European vanilla call options available in the market are given by

$$
C_{i}[K]=\mathbb{E}\left[D\left(T_{i}\right)\left(X_{i}-K\right)_{+}\right], \quad i=1, \ldots, n
$$

In the following sections, we will first study two classical examples of exotic options, namely basket and Asian options, in the stochastic interest rate environment. Afterwards, we will derive the optimal strategy in the framework (1) and apply similar techniques to some other exotic products with more complex pay-offs. 


\subsubsection{Basket option case}

For a basket option, $S$ stands for $\sum_{i=1}^{n} w_{i} X_{i}=\sum_{i=1}^{n} w_{i} S_{i}(T)$, where $S_{i}(T)$ denotes the price level of stock $i$ at time $T$. Inequality (1) provides

$$
\left(\sum_{i=1}^{n} w_{i} S_{i}(T)-K\right)_{+} \leq \sum_{i=1}^{n} w_{i}\left(S_{i}(T)-K_{i}\right)_{+}
$$

when $\sum_{i=1}^{n} w_{i} K_{i} \leq K$ and $K_{i} \geq 0, i=1, \ldots, n$.

The right-hand side of inequality (41) can be interpreted as the pay-off at time $T$ of a strategy consisting of buying at time zero a number of $w_{i}$ European options with pay-off $\left(S_{i}(T)-K_{i}\right)_{+}$ at time $T, i=1, \ldots, n$, holding these options until they expire at time $T$ and exercising the ones with positive pay-offs. Since the pay-off of such a strategy dominates the pay-off of the exotic option according to inequality (41), it is a super-replicating strategy. The price of this strategy at time zero is given by $\sum_{i=1}^{n} w_{i} C_{i}\left[K_{i}\right]$. From 40, , we have that

$$
\sum_{i=1}^{n} w_{i} C_{i}\left[K_{i}\right]=\sum_{i=1}^{n} w_{i} \mathbb{E}\left[D(T)\left(S_{i}(T)-K_{i}\right)_{+}\right]
$$

By taking for each $i, i=1, \ldots, n, \zeta=\zeta_{i}=\frac{D(T)}{P(0, T)}$, we can write 42 as

$$
\sum_{i=1}^{n} w_{i} \mathbb{E}\left[D(T)\left(S_{i}(T)-K_{i}\right)_{+}\right]=P(0, T) \sum_{i=1}^{n} w_{i} \mathbb{E}\left[\zeta\left(S_{i}(T)-K_{i}\right)_{+}\right]
$$

Then the optimal strikes, $K_{i}, i=1, \ldots, n$ corresponding to the cheapest super-replicating strategy follow from the corresponding optimization problem $(28)$ under the $\mathbb{Q}$-measure. 


\subsubsection{Asian option case}

Now we take $\sum_{i=1}^{n} w_{i} X_{i}=\sum_{i=1}^{n} w_{i} S\left(T_{i}\right)$, where $S\left(T_{i}\right)$ denotes the price level of a stock or index at time $T_{i}$. Similarly to the basket option case, we have that

$$
\left(\sum_{i=1}^{n} w_{i} S\left(T_{i}\right)-K\right)_{+} \leq \sum_{i=1}^{n} w_{i}\left(S\left(T_{i}\right)-K_{i}\right)_{+}
$$

holds when $\sum_{i=1}^{n} w_{i} K_{i} \leq K$ and $K_{i} \geq 0, i=1, \ldots, n$.

The right-hand side of inequality (43) can be interpreted as the pay-off at time $T$ of a strategy of buying at time zero $w_{i}$ exchange options with pay-off $\left(S\left(T_{i}\right) P\left(T_{i}, T\right)-K_{i} P\left(T_{i}, T\right)\right)_{+}$at time $T_{i}$, holding these options until they expire at time $T_{i}, i=1, \ldots, n$, exercising the ones with positive pay-offs and investing the pay-offs for the period $\left[T_{i}, T\right]$ by buying $S\left(T_{i}\right)-K_{i}$ zerocoupon bonds at a price $P\left(T_{i}, T\right)$. We introduce $C_{S\left(T_{i}\right) P\left(T_{i}, T\right)}\left[K_{i} P\left(T_{i}, T\right)\right]$ to denote the price at time zero of an exchange option with maturity $T_{i}$ and pay-off $\left(S\left(T_{i}\right) P\left(T_{i}, T\right)-K_{i} P\left(T_{i}, T\right)\right)_{+}$at time $T_{i}$.

As the pay-off of the exchange option indicates, the buyer of the option has the right to obtain at time $T_{i}$ the difference between 'the value of the stock times a zero-coupon bond with maturity $T$ ' and 'the strike $K_{i}$ times a zero-coupon bond with maturity $T$ '. Also, since the pay-off of the strategy dominates the pay-off of the exotic option according to inequality (43), we have found a super-replicating strategy. The price of this strategy is $\sum_{i=1}^{n} w_{i} C_{S\left(T_{i}\right) P\left(T_{i}, T\right)}\left[K_{i} P\left(T_{i}, T\right)\right]$.

The time- 0 price of the derivative which pays $\left(S\left(T_{i}\right)-K_{i}\right)_{+}$at time $T$ is equal to $\mathbb{E}\left[D(T)\left(S\left(T_{i}\right)-K_{i}\right)_{+}\right]$. Such a contract can be hedged by investing at time $t=0$ in a derivative which pays $\left(S\left(T_{i}\right) P\left(T_{i}, T\right)-K_{i} P\left(T_{i}, T\right)\right)_{+}$at time $T_{i}$, and investing the eventual pay-off at time $T_{i}$ in zero-coupon bonds until time $T$. The price of this hedging strategy is given by $C_{S\left(T_{i}\right) P\left(T_{i}, T\right)}\left[K_{i} P\left(T_{i}, T\right)\right]$ and must be equal to the price of the derivative, which is $\mathbb{E}\left[D(T)\left(S\left(T_{i}\right)-K_{i}\right)_{+}\right]$. We then find that

$$
\sum_{i=1}^{n} w_{i} C_{S\left(T_{i}\right) P\left(T_{i}, T\right)}\left[K_{i} P\left(T_{i}, T\right)\right]=\sum_{i=1}^{n} w_{i} \mathbb{E}\left[D(T)\left(S\left(T_{i}\right)-K_{i}\right)_{+}\right]
$$


Similarly as for the basket option case, we take for each $i, i=1, \ldots, n, \zeta=\zeta_{i}=\frac{D(T)}{P(0, T)}$ and we write (44) as

$$
\sum_{i=1}^{n} w_{i} \mathbb{E}\left[D(T)\left(S\left(T_{i}\right)-K_{i}\right)_{+}\right]=P(0, T) \sum_{i=1}^{n} w_{i} \mathbb{E}\left[\zeta\left(S\left(T_{i}\right)-K_{i}\right)_{+}\right] .
$$

Then the optimal strikes, $K_{i}, i=1, \ldots, n$, follow from the corresponding optimization problem (28) under the $\mathbb{Q}$-measure.

In a deterministic interest rate setting, the bond price $P\left(T_{i}, T\right)$ is known at time 0 and as a result we can buy the optimal super-hedging portfolio for an Asian option with payoff $\left(\sum_{i=1}^{n} w_{i} S\left(T_{i}\right)-K\right)_{+}$by investing in vanilla call options with expiration dates $T_{i}, i=1,2, \ldots, n$. When interest rates behave in a stochastic way, $P\left(T_{i}, T\right)$ is not known at time 0 and the optimal portfolio consists of exchange options with prices $C_{S\left(T_{i}\right) P\left(T_{i}, T\right)}\left[K_{i} P\left(T_{i}, T\right)\right], i=1,2, \ldots, n$. In Chen et al. (2008), it is assumed that we can only invest in a deterministic number of vanilla calls which expire at $T_{i}, i=1,2, \ldots, n$ in order to construct a super-hedging portfolio. Constructing the optimal, model-free, static super-replicating portfolio in a stochastic interest rate setting, however, requires a market where derivatives with payoff $\left(S\left(T_{i}\right) P\left(T_{i}, T\right)-K_{i} P\left(T_{i}, T\right)\right)_{+}$at time $T_{i}$ are traded. This means that in a stochastic interest rate setting, the minimal price may not be attainable in the class of all super-replicating strategies which consist of only vanilla call options.

\subsubsection{Floating strike Asian option}

The optimization method described above can also be applied to other derivatives with more complex pay-offs. A first option that we consider is a floating strike Asian option.

The pay-off of the floating strike Asian option, as discussed in Vanmaele et al. (2006), is given by

$$
\left(\sum_{i=1}^{n} w_{i} S(T-i+1)-\beta S(T)\right)_{+}=S(T)\left(\sum_{i=1}^{n} w_{i} \frac{S(T-i+1)}{S(T)}-\beta\right)_{+}
$$


with $S(T)>0$ and $\beta$ a positive percentage and $w_{i}=\frac{1}{n}, i=1, \ldots, n$. Further we have that

$$
\begin{aligned}
\left(\sum_{i=1}^{n} w_{i} S(T-i+1)-\beta S(T)\right)_{+} & \leq \sum_{i=1}^{n} w_{i}\left(S(T-i+1)-S(T) K_{i}\right)_{+} \\
& =\sum_{i=1}^{n} w_{i} K_{i}\left(\frac{S(T-i+1)}{K_{i}}-S(T)\right)_{+}
\end{aligned}
$$

with $\sum_{i=1}^{n} w_{i} K_{i} \leq \beta$ and $K_{i} \geq 0, i=1, \ldots, n$. The right-hand side of inequality 45 can be interpreted as the pay-off at time $T$ of a strategy consisting of buying at time zero $w_{i} K_{i}$ forward start put options with pay-off $\left(\frac{S(T-i+1)}{K_{i}}-S(T)\right)_{+}$at time $T, i=1, \ldots, n$, holding these options until they expire at time $T$ and exercising the ones with positive pay-offs.

A forward start put option with pay-off $\left(\frac{S(T-i+1)}{K_{i}}-S(T)\right)_{+}$is a vanilla put option, but with a variable strike given by a percentage of $S(T-i+1)$ which is only known from $T-i+1$ on. For more information about forward start options we refer to Weber and Wystup (2009).

Since the pay-offs of these strategies dominate the pay-off of the exotic option according to the inequality (45), they are super-replicating strategies. The prices of these strategies are

$$
\begin{aligned}
& \sum_{i=1}^{n} w_{i} \mathbb{E}\left[D(T)\left(S(T-i+1)-S(T) K_{i}\right)_{+}\right] \\
& =\sum_{i=1}^{n} w_{i} \mathbb{E}\left[D(T) S(T)\left(\frac{S(T-i+1)}{S(T)}-K_{i}\right)_{+}\right]
\end{aligned}
$$

Because $\{D(t) S(t), t \geq 0\}$ is a martingale under $\mathbb{Q}$, it suffices to take $\zeta_{i}=\frac{D(T) S(T)}{S(0)}$ for all $i=1, \ldots, n$. The optimal $K_{i}, i=1, \ldots, n$, satisfying $\sum_{i=1}^{n} w_{i} K_{i} \leq \beta$, which lead to the least price among these strategies can then be determined via the procedure explained in Section 3.1 .

\subsubsection{Option struck in foreign currency}

As another example of more complex derivatives, we consider options struck in foreign currency (see e.g. Musiela and Rutkowski (2005) p.176), with the underlying $\mathbb{S}^{f}$ either a weighted average of different asset prices or a weighted average of asset prices at different dates and the strike 
$K^{f}$ also expressed in the foreign currency. Denote the exchange rate process by $\{Q(t), t \geq 0\}$. Hereafter, we will only consider the case where $\mathbb{S}^{f}$ is a weighted average of different asset prices.

Denote $\mathbb{S}^{f}$ for $\sum_{i=1}^{n} w_{i} S_{i}^{f}(T)$ with $S_{i}^{f}(T)$ the equity price of asset $i$ in the foreign currency and $w_{i}, i=1, \ldots, n$, the positive weight factors. The pay-off at time $T$ in the domestic currency of a foreign basket call struck in foreign currency equals $Q(T)\left(\mathbb{S}^{f}-K^{f}\right)_{+}$.

Further we have that

$$
\left(\mathbb{S}^{f}-K^{f}\right)_{+}=\left(\sum_{i=1}^{n} w_{i} S_{i}^{f}(T)-K^{f}\right)_{+} \leq \sum_{i=1}^{n} w_{i}\left(S_{i}^{f}(T)-K_{i}^{f}\right)_{+}
$$

with $\sum_{i=1}^{n} w_{i} K_{i}^{f} \leq K^{f}$ and $K_{i}^{f} \geq 0, i=1, \ldots, n$, and since $Q(T)>0$,

$$
Q(T)\left(\mathbb{S}^{f}-K^{f}\right)_{+} \leq \sum_{i=1}^{n} w_{i} Q(T)\left(S_{i}^{f}(T)-K_{i}^{f}\right)_{+}
$$

The right-hand side of inequality (46) can be interpreted as the pay-off at time $T$ of a strategy consisting of buying in the domestic currency at time zero $w_{i}$ foreign call options struck in foreign currency with pay-off $\left(S_{i}^{f}(T)-K_{i}^{f}\right)_{+}$at time $T, i=1, \ldots, n$, holding these options until they expire at time $T$ and exercising the ones with positive pay-offs. These strategies super-replicate the foreign basket call struck in foreign currency and exchanged into the domestic currency. Their prices under the domestic martingale measure are given by

$$
\sum_{i=1}^{n} w_{i} \mathbb{E}\left[D(T) Q(T)\left(S_{i}^{f}(T)-K_{i}^{f}\right)_{+}\right]
$$

If we take $\zeta_{i}=\frac{D(T) Q(T)}{\mathbb{E}[D(T) Q(T)]}, i=1, \ldots, n$, the optimal $K_{i}^{f}, i=1, \ldots, n$, leading to the least price among these strategies can be determined via the optimization procedure explained in Section 3.1 .

We note that the stochastic factors $\zeta_{i}=\zeta, i=1, \ldots, n$, define a change of measure as in the proof of Lemma 3. For the example of the basket option and the Asian option $\zeta$ defines the $T$-forward measure while for the floating strike Asian option it defines the martingale measure associated 
with the numeraire $S$.

\section{Is the optimal solution consistent with no-arbitrage?}

The price of the optimal static super-replicating strategy in Hobson et al. (2005) and Chen et al. (2008) is an upper bound for the exotic option price. It is reached when the underlying random variables are comonotonic. Hereafter, we assume the existence of an equivalent martingale measure, which is 'essentially' equal to the no-arbitrage condition, and we investigate the question whether the comonotonicity property can co-exist with the existence of this equivalent martingale measure. If yes, the price of the strategy is a reachable upper bound of the exotic option. If not, it is an unreachable upper bound. Two situations have to be investigated. Firstly, different assets are comonotonic. Secondly, prices of one asset at different time points are comonotonic. These two situations correspond to the basket option case and the Asian option case respectively.

\subsection{Several underlying assets}

In Dhaene et al. (2013) the following reasoning is made to show that the comonotonicity property cannot co-exist with the martingale property in certain situations.

Let $S_{1}(t)$ and $S_{2}(t)$ denote the prices of two underlying assets at time $t, t=0,1,2, \ldots, T$. For simplicity, the risk-free interest rate $r$ is assumed to be zero. Further, consider an increasing function $f:(0,+\infty) \rightarrow(0,+\infty)$. When $S_{2}(t)=f\left(S_{1}(t)\right)$, the random variables $S_{1}(t)$ and $S_{2}(t)$ are comonotonic for each time point $t$. For time $t_{1}<t_{2}$, according to the martingale property, we have that

$$
\mathbb{E}\left[S_{l}\left(t_{2}\right) \mid S_{l}\left(t_{1}\right)\right]=S_{l}\left(t_{1}\right), \quad l=1,2 .
$$

Now suppose $f$ is a strictly convex function. If the conditional distribution of $S_{1}\left(t_{2}\right)$, given $S_{1}\left(t_{1}\right)$ is not degenerate, according to the strict convexity of $f$ and Jensen's inequality, we can 
get

$$
f\left(S_{1}\left(t_{1}\right)\right)=\mathbb{E}\left[f\left(S_{1}\left(t_{2}\right)\right) \mid S_{1}\left(t_{1}\right)\right]>f\left(\mathbb{E}\left[S_{1}\left(t_{2}\right) \mid S_{1}\left(t_{1}\right)\right]\right)=f\left(S_{1}\left(t_{1}\right)\right),
$$

and thus

$$
f\left(S_{1}\left(t_{1}\right)\right)>f\left(S_{1}\left(t_{1}\right)\right) .
$$

This is a contradiction. Therefore, the comonotonicity property cannot co-exist with the martingale property in this situation. Notice that if $f$ is linear, the comonotonicity property and the martingale property might co-exist.

\subsection{A single underlying asset}

\subsubsection{Some definitions}

In order to investigate the co-existence of the comonotonicity property and the martingale property for one underlying asset case, we extend some definitions from Dhaene et al. (2002a) to the notion of strict comonotonicity.

Definition 5 A subset $A \subseteq \mathbb{R}^{n}$ is called a support of an $n$-dimensional random vector $\underline{X}=$ $\left(X_{1}, \ldots, X_{n}\right)$ if $\mathbb{P}[\underline{X} \in A]=1$ holds true.

An $n$-vector $\left(x_{1}, x_{2}, \ldots, x_{n}\right)$ will be denoted by $\underline{x}$. For two $n$-vectors $\underline{x}$ and $\underline{y}$, the notation $\underline{x} \leq \underline{y}$ will be used for the componentwise order which is defined by $x_{i} \leq y_{i}$ for all $i=1,2, \ldots, n$ and the notation $\underline{x}<\underline{y}$ will be used for the componentwise order which is defined by $x_{i}<y_{i}$ for all $i=1,2, \ldots, n$.

Definition 6 The set $A \subseteq \mathbb{R}^{n}$ is said to be comonotonic iffor any $\underline{x}$ and $\underline{y}$ in $A$, either $\underline{x} \leq \underline{y}$ or $\underline{y} \leq \underline{x}$ holds. The set $A$ is said to be strictly comonotonic if for any $\underline{x}$ and $\underline{y}$ (different from $\underline{x}$ ) in A, either $\underline{x}<\underline{y}$ or $\underline{y}<\underline{x}$ holds. 

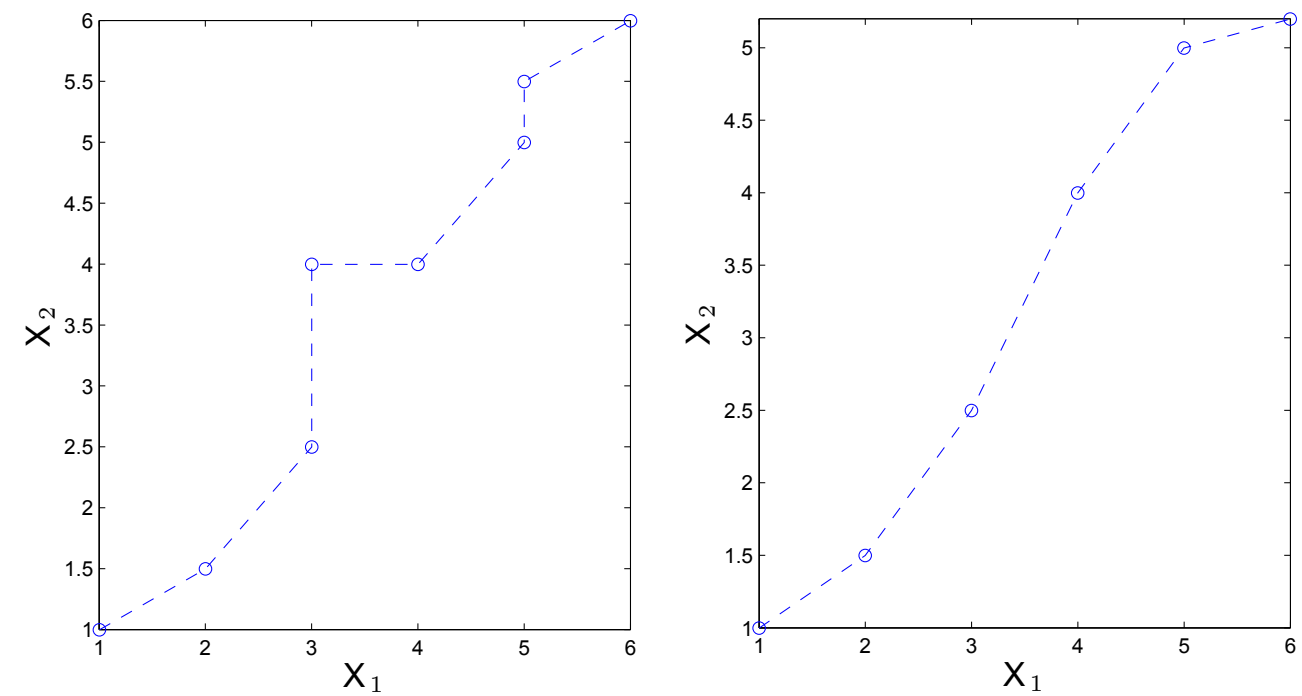

Figure 3: Comonotonic support (left panel) for a couple $\left(X_{1}, X_{2}\right)$ and a strict comonotonic support (right panel) for a couple $\left(X_{1}, X_{2}\right)$.

Definition 7 A random vector $\underline{X}=\left(X_{1}, \ldots, X_{n}\right)$ is said to be comonotonic if it has a comonotonic support. It is said to be strictly comonotonic if it has a strictly comonotonic support.

Combining the definitions above, it holds that a random vector $\underline{X}$ is comonotonic if there exists a comonotonic set $A \subseteq \mathbb{R}^{n}$ such that $\mathbb{P}[\underline{X} \in A]=1$. A strictly comonotonic $\underline{X}$ means that there exists a strictly comonotonic set $A \subseteq \mathbb{R}^{n}$ such that $\mathbb{P}[\underline{X} \in A]=1$. Definition 7 is illustrated in Figure 3. In this figure, we show the support of a comonotonic and a strict comonotonic random couple. A possible comonotonic situation which is not strictly comonotonic is illustrated in the left panel of Figure 3. An example of a strictly comonotonic support is shown in the right panel of the same figure. Obviously horizontal and vertical line segments are not allowed for strict comonotonicity.

In the following lemma, we show that comonotonicity and strict comonotonicity of a random vector $\underline{X}$ are equivalent when the marginals are continuous.

Lemma 8 Consider a random vector $\underline{X}=\left(X_{1}, \ldots, X_{n}\right)$ with continuous marginal cdf's $F_{X_{i}}$, 
$i=1,2, \ldots n$. Then we have that

$\underline{X}$ is comonotonic $\Leftrightarrow \underline{X}$ is strictly comonotonic.

Proof. The proof of the 'converse' part is trivial.

In order to prove the 'direct' part, assume that $\underline{X}$ is comonotonic. A support for the random vector $\underline{X}$ is given by

$$
\operatorname{support}[\underline{X}]=\left\{\left(F_{X_{1}}^{-1}(p), F_{X_{2}}^{-1}(p), \ldots, F_{X_{n}}^{-1}(p)\right) \mid 0<p<1\right\} \text {. }
$$

We can take any $\underline{x}, \underline{y} \in \operatorname{support}[\underline{X}]$. Then there exist values $p_{1}, p_{2} \in(0,1)$ such that $\underline{x}=$ $\left(F_{X_{1}}^{-1}\left(p_{1}\right), F_{X_{2}}^{-1}\left(p_{1}\right), \ldots, F_{X_{n}}^{-1}\left(p_{1}\right)\right)$ and $\underline{y}=\left(F_{X_{1}}^{-1}\left(p_{2}\right), F_{X_{2}}^{-1}\left(p_{2}\right), \ldots, F_{X_{n}}^{-1}\left(p_{2}\right)\right)$. If $p_{1}=p_{2}$, we obviously have that $\underline{x}=\underline{y}$. Let us now consider the situation where $p_{1}<p_{2}$. We will show that this implies that $\underline{x}<\underline{y}$ must hold. As $F_{X_{i}}^{-1}$ is non-decreasing, we have that $\underline{x} \leq \underline{y}$. Because $F_{X_{i}}$ is continuous on $(0,1)$, we have that $F_{X_{i}}^{-1}$ is strictly increasing on $(0,1)$ which implies that $F_{X_{i}}^{-1}\left(p_{1}\right)=F_{X_{i}}^{-1}\left(p_{2}\right)$ can only be satisfied when $p_{1}=p_{2}$. We conclude that $\underline{x}<\underline{y}$ must hold.

Similarly, starting from $p_{1}>p_{2}$ it follows that $\underline{x}>\underline{y}$, from which we conclude that $\underline{X}$ is strictly comonotonic.

The assumption that the marginal cdf's must be continuous is not too restrictive. In case the dynamics of the stock price process $\{S(t) \mid 0 \leq t \leq T\}$ are described by the Black-Scholes model (or Variance Gamma, Heston,...), the cdf $F_{S(t)}$ of the price $S(t), t>0$, is continuous. In the following section we investigate the martingale property and the (strict) comonotonicity property of the random vector $\underline{X}$.

\subsection{2 (Strict) comonotonicity and martingale property}

Theorem 9 For prices $S\left(t_{i}\right)$ of a given asset at times $t_{i}, i=1,2, \ldots, n$ with $t_{1}<t_{2}<\ldots<t_{n}$, denote $\underline{S}=\left(S\left(t_{1}\right), \ldots, S\left(t_{n}\right)\right)$. If $\underline{S}$ is a strictly comonotonic vector and the martingale property 
holds, then for $i<j$, we have that $S\left(t_{i}\right)$ and $S\left(t_{j}\right)$ are related through the linear relationship $S\left(t_{j}\right)=S\left(t_{i}\right) e^{r\left(t_{j}-t_{i}\right)}$ almost surely.

Proof. If $\underline{S}$ is strictly comonotonic, we have that $\left(S\left(t_{i}\right), S\left(t_{j}\right)\right)$ is strictly comonotonic for any $i<j$. Since $\left(S\left(t_{i}\right), S\left(t_{j}\right)\right)$ is a strictly comonotonic vector, $\left(S\left(t_{i}\right), S\left(t_{j}\right)\right)$ has a strictly comonotonic support.

Similar to $X_{1}$ and $X_{2}$ in the right panel of Figure $3, S\left(t_{i}\right)$ and $S\left(t_{j}\right)$ are paired in such a way that, if $S\left(t_{i}\right)$ is given, there exists a corresponding $S\left(t_{j}\right)$ or in another form

$$
\mathbb{E}\left[S\left(t_{j}\right) \mid S\left(t_{i}\right)\right]=S\left(t_{j}\right) \quad \text { almost surely. }
$$

Also, according to the martingale property, we have

$$
\mathbb{E}\left[S\left(t_{j}\right) \mid S\left(t_{i}\right)\right]=S\left(t_{i}\right) \mathrm{e}^{r\left(t_{j}-t_{i}\right)}
$$

We conclude that

$$
S\left(t_{j}\right)=S\left(t_{i}\right) \mathrm{e}^{r\left(t_{j}-t_{i}\right)}, \text { a.s. }
$$

Note that under the assumptions of Theorem 9 the stock price process evolves as a risk free asset.

A natural question is what happens when the assumption of strict comonotonicity is relaxed to comonotonicity? In Remark 10 below, we show by an example that when $\underline{S}$ is a comonotonic, but not a strictly comonotonic vector, the comonotonicity property and the martingale property can co-exist without the linear relationship 47).

Remark 10 (A counter example) Let us assume that $r=0$ without loss of generality, which means $e^{r\left(t_{j}-t_{i}\right)}=1$. Furthermore, suppose that the possible outcomes of $\underline{S}$ are $(1,0.5),(1,1.5)$, $(3,2.5)$ and $(3,3.5)$ with probability 0.25 for each outcome under the equivalent martingale mea- 
sure. The martingale property

$$
\mathbb{E}\left[S\left(t_{j}\right) \mid S\left(t_{i}\right)\right]=S\left(t_{i}\right) \mathrm{e}^{r\left(t_{j}-t_{i}\right)}=S\left(t_{i}\right)
$$

holds in this case. Indeed, we find that

$$
\mathbb{E}\left[S\left(t_{j}\right) \mid S\left(t_{i}\right)=1\right]=1
$$

and

$$
\mathbb{E}\left[S\left(t_{j}\right) \mid S\left(t_{i}\right)=3\right]=3
$$

In addition, it is easy to see that $\underline{S}$ has a comonotonic but not strictly comonotonic support, so $\underline{S}$ is a comonotonic vector. As a result, the comonotonicity property and the martingale property can co-exist without the linear relationship (47).

\subsubsection{The Black-Scholes model and option price curve}

We can take a further look at the situation where the stock price process $\{S(t) \mid 0 \leq t \leq T\}$ is described by a stochastic process with continuous cdf's $F_{S(t)}$ for all $t>0$. Let us concentrate on the Black-Scholes model. Suppose that the prices $S\left(t_{i}\right)$ and $S\left(t_{j}\right)$ at time $t_{i}$ and $t_{j}$ with $0<t_{i}<t_{j} \leq T$ are comonotonic. Since $F_{S(t)}$ is continuous, by Lemma 8 we have that $S\left(t_{i}\right)$ and $S\left(t_{j}\right)$ are strictly comonotonic. Therefore the vector $\left(S\left(t_{i}\right), S\left(t_{j}\right)\right)$ has a strictly comonotonic support $A$ with

$$
A=\left\{\left(F_{S\left(t_{i}\right)}^{-1}(p), F_{S\left(t_{j}\right)}^{-1}(p)\right) \mid 0<p<1\right\}
$$

This means that knowing $S\left(t_{i}\right)$ implies knowing $S\left(t_{j}\right)$ and vice versa. However in the BlackScholes model, one price is not fully determined by the other due to independent log increments. We can conclude that comonotonicity cannot hold in this particular stock price model: there is an inconsistency between the Black-Scholes model and the comonotonicity assumption of $S\left(t_{i}\right)$ and $S\left(t_{j}\right)$. 
Let us continue with the assumptions above that $\left(S\left(t_{i}\right), S\left(t_{j}\right)\right)$ is comonotonic and $F_{S(t)}$ is continuous for all $t>0$. In addition we assume that the martingale property holds. Then from Lemma 8 and Theorem 9 we conclude that comonotonicity of the vector $\left(S\left(t_{i}\right), S\left(t_{j}\right)\right)$ can only co-exist with the martingale property if the linear relationship (47) holds.

Clearly, in the Black-Scholes model the martingale property holds but (47) is not valid, implying that the vector $\left(S\left(t_{i}\right), S\left(t_{j}\right)\right)$ cannot be strictly comonotonic. The conflict between comonotonicity and the Black-Scholes model is then due to the continuity of the cdf's $F_{S(t)}$.

From here on, we relax the condition that the cdf's $F_{S(t)}$ are continuous and only assume that the vector $\left(S\left(t_{i}\right), S\left(t_{j}\right)\right)$ is strictly comonotonic and that the martingale property holds. By applying Theorem 9, we derive a relation similar to the relationship (47) but now between the cdf's of $S\left(t_{i}\right)$ and $S\left(t_{j}\right)$ :

$$
F_{S\left(t_{i}\right)}(s)=F_{S\left(t_{j}\right)}\left(s \mathrm{e}^{r\left(t_{j}-t_{i}\right)}\right) .
$$

Using expression (12), this relation can be rewritten as

$$
F_{S\left(t_{j}\right)}^{-1\left(\alpha_{i, j}\right)}\left(F_{S\left(t_{i}\right)}(s)\right)=s \mathrm{e}^{r\left(t_{j}-t_{i}\right)}
$$

for some $\alpha_{i, j} \in[0,1]$.

In the next theorem, we prove that under the assumptions of Theorem 9 , namely strict comonotonicity of $\left(S\left(t_{1}\right), \ldots, S\left(t_{n}\right)\right)$ and the martingale property, options with maturity $t_{j}$ can be determined using the option curve with maturity $t_{i}, i \neq j$. The price of a European call option on the stock $S$, with strike $K$ and maturity $T$ is denoted by $C[K, T]$.

Theorem 11 Let us denote $\underline{S}=\left(S\left(t_{1}\right), \ldots, S\left(t_{n}\right)\right)$ with $S\left(t_{i}\right)$ being the price of the underlying asset at time $t_{i}, i=1,2, \ldots, n$ with $t_{1}<t_{2}<\ldots<t_{n}$. If $\underline{S}$ is a strictly comonotonic vector and the martingale property holds, then

$$
C\left[K, t_{j}\right]=C\left[\mathrm{e}^{-r\left(t_{j}-t_{i}\right)} K, t_{i}\right], \quad i, j=1,2, \ldots, n
$$


Proof. If $\underline{S}$ is a strictly comonotonic vector, then we find that for any $i \neq j,\left(S\left(t_{i}\right), S\left(t_{j}\right)\right)$ is also strictly comonotonic.

Take any $i \in\{1,2, \ldots, n\}$. Then by the martingale property we can invoke relation (47) of Theorem 9 leading to the following equalities for any $i \neq j$ and any $K$ :

$$
\begin{aligned}
C\left[K, t_{j}\right] & =e^{-r t_{j}} \mathbb{E}\left[\left(S\left(t_{j}\right)-K\right)_{+}\right] \\
& \stackrel{47}{=} e^{-r t_{j}} \mathbb{E}\left[\left(S\left(t_{i}\right) e^{r\left(t_{j}-t_{i}\right)}-K\right)_{+}\right] \\
& =e^{-r t_{i}} \mathbb{E}\left[\left(S\left(t_{i}\right)-e^{-r\left(t_{j}-t_{i}\right)} K\right)_{+}\right] \\
& =C\left[e^{-r\left(t_{j}-t_{i}\right)} K, t_{i}\right],
\end{aligned}
$$

which proves the result.

Relation (49) puts a restriction on the option price surface, which for given prices may not be satisfied in practice. In these situations, we have to conclude that $\underline{S}$ cannot be a comonotonic vector.

In case the vector $\underline{S}$ is strictly comonotonic and the martingale property holds, we can use (47) of Theorem 9 to write the price of an Asian option with maturity $T$ and strike $K$ as follows, and for any $i \in\{1,2, \ldots, n\}$ :

$$
\mathrm{e}^{-r T} \mathbb{E}\left[\left(\frac{\sum_{k=1}^{n} S\left(t_{k}\right)}{n}-K\right)_{+}\right]=\frac{\mathrm{e}^{-r T}}{n} \mathbb{E}\left[\left(S\left(t_{i}\right) \sum_{k=1}^{n} \mathrm{e}^{r\left(t_{k}-t_{i}\right)}-n K\right)_{+}\right],
$$

which results in

$$
\mathrm{e}^{-r T} \mathbb{E}\left[\left(\frac{\sum_{k=1}^{n} S\left(t_{k}\right)}{n}-K\right)_{+}\right]=\frac{\mathrm{e}^{-r T}}{n} c_{i} \mathbb{E}\left[\left(S\left(t_{i}\right)-\frac{n K}{c_{i}}\right)_{+}\right],
$$

where $c_{i}=\sum_{k=1}^{n} e^{r\left(t_{k}-t_{i}\right)}$. So we find that the price of an Asian option in an arbitrage-free market can be calculated in this strictly comonotonic setting by using only the price of a European call option which expires at $t_{i}$. The behavior of the asset $S$ at time $t_{i}$ determines the behavior of the asset $S$ at the other time points $j \neq i$. 
Note that in Albrecher et al. (2008) it is already shown that in case the vector $\underline{S}=\left(S\left(t_{1}\right), \ldots, S\left(t_{n}\right)\right)$ has continuous marginals, comonotonicity of $\underline{S}$ together with the martingale property implies that (48) must hold with $\alpha_{i, j}=1$. In Theorem 9 we derived a linear relationship which has to hold and this is stronger than the conclusion regarding cdf's. Additionally Theorem 9 only requires that $\left(S\left(t_{i}\right), S\left(t_{j}\right)\right)$ is strictly comonotonic and hence compared to the conclusion in Albrecher et al. (2008), no continuity of $F_{S(t)}$ is required. We further notice that Albrecher et al. (2008) derived relation (49) in another way for the situation where the marginals $S\left(t_{i}\right)$ have continuous cdf's.

\subsection{Discussion}

For basket options, the pay-off is determined by the weighted sum of several underlying assets prices at maturity time $T$. As it is shown in Section 4.1, for the case with several underlying assets, there is a contradiction between the martingale property and the comonotonicity property at each time point $t=0,1,2, \ldots, T$. However, when we study the comonotonicity property for the underlying assets of basket options, we do not require that $S_{2}(t)=f\left(S_{1}(t)\right)$ for an increasing and strictly convex function $f$. Instead, only comonotonicity of $\left(S_{1}(t), S_{2}(t)\right)$ is assumed. So we cannot conclude from Section 4.1 that there is a contradiction between the comonotonicity property and the martingale property for the basket options case and a further study is needed.

For Asian options, the pay-off is determined by the weighted sum of the underlying price over some pre-set period of time. According to Section 4.2 , when there is only one underlying asset, we know that the co-existence of the strict comonotonicity property and the martingale property implies the linear relationship (47) of Theorem 9 and the restriction (49) of Theorem 11 on the European call option price surface. However, it is also possible that there is a contradiction between them, as e.g. in the Black-Scholes model. When the comonotonicity property contradicts the martingale property, comonotonic underlying random variables are impossible in the market. In this case, the price of the optimal static super-replicating strategies as an upper bound may not be reached by the Asian option price, since it can only be reached by the Asian option price when the underlying random variables are comonotonic. 


\section{Conclusion}

In this paper, we investigated an optimization problem related to super-replicating strategies for European-type call options written on a weighted sum $S=w_{1} X_{1}+\cdots+w_{n} X_{n}$ of asset prices. Firstly, we proved that in general, the optimal solution is non-unique. This observation is useful since it allows some flexibility to compose the optimal super-replicating strategies in a real market situation, which often has some constraints in trading. Secondly, a generalized optimization problem with random weights has been studied. Using these results, we derived optimal static super-replicating strategies for different kind of options in a stochastic interest rate setting. Thirdly, the co-existence of the comonotonicity property and the martingale property was studied. We have seen that in the case of a single underlying asset they can co-exist, for instance as it was shown in Remark 10. However if the price vector of the underlying asset is strictly comonotonic, a linear relationship of the underlying prices has to hold and therefore the comonotonicity property and the martingale property can lead to a contradiction. So for Asian options e.g., the price of the optimal static super-replicating strategies may be strictly larger than the Asian option price.

\section{References}

Albrecher, H., Mayer, P. A. and Schoutens, W. (2008), 'General lower bounds for arithmetic asian option prices', Applied Mathematical Finance 15(2), 123-149.

Chen, X., Deelstra, G., Dhaene, J. and Vanmaele, M. (2008), 'Static super-replicating strategies for a class of exotic options', Insurance: Mathematics \& Economics 42(3), 1067-1085.

Cheung, K. C., Dhaene, J., Rong, P. and Yam, P. S. C. (2013), Explicit solutions for a general class of optimal allocation problems, Research report AFI-1378 FEB, KU Leuven.

Deelstra, G., Dhaene, J. and Vanmaele, M. (2011), An overview of comonotonicity and its applications in finance and insurance, in B. Oksendal and G. Nunno, eds, 'Advanced Mathematical Methods for Finance', Springer Berlin Heidelberg, pp. 155-179. 
Dhaene, J., Denuit, M., Goovaerts, M., Kaas, R. and Vyncke, D. (2002a), 'The concept of comonotonicity in actuarial science and finance: theory', Insurance: Mathematics \& Economics 31(1), 3-33.

Dhaene, J., Denuit, M., Goovaerts, M., Kaas, R. and Vyncke, D. (2002b), 'The concept of comonotonicity in actuarial science and finance: applications', Insurance: Mathematics \& Economics 31(2), 133-161.

Dhaene, J., Goovaerts, M. and Kaas, R. (2003), 'Economic capital allocation derived from risk measures', North American Actuarial Journal 7, 44-59.

Dhaene, J. and Kukush, A. (2011), 'Comonotonic modification of random vector in its own probability space', Research report AFI-1151, FEB, K.U.Leuven .

Dhaene, J., Kukush, A. and Linders, D. (2013), Comonotonic asset prices in arbitrage-free markets, Working Paper, Leuven: KU Leuven - Faculty of Business and Economics.

Dhaene, J., Linders, D., Schoutens, W. and Vyncke, D. (2012), 'The herd behavior index: A new measure for the implied degree of co-movement in stock markets', Insurance: Mathematics and Economics 50(3), 357-370.

Dhaene, J., Tsanakas, A., Valdez, E. A. and Vanduffel, S. (2012), 'Optimal capital allocation principles', Journal of Risk and Insurance 79(1), 1-28.

Furman, E. and Zitikis, R. (2008), 'Weighted risk capital allocations', Insurance: Mathematics and Economics 43(2), 263 - 269.

Geman, H., Karoui, N. E. and Rochet, J. (1995), 'Change of Numeraire, Changes of Probability Measures and Pricing of Options', Jornal of Applied Probability 32, 443-458.

Hobson, D., Laurence, P. and Wang, T. (2005), 'Static-arbitrage upper bounds for the prices of basket options', Quantitative Finance 5(4), 329-342. 
Laeven, R. J. and Goovaerts, M. J. (2004), 'An optimization approach to the dynamic allocation of economic capital', Insurance: Mathematics and Economics 35(2), 299 - 319.

Linders, D., Dhaene, J., Hounnon, H. and Vanmaele, M. (2012), Index options: a model-free approach, Research report afi-1265 feb, Leuven: KU Leuven - Faculty of Business and Economics.

Musiela, M. and Rutkowski, M. (2005), Martingale methods in financial modeling, SpringerVerlag, Berlin. 2nd edition.

Nielsen, J. and Sandman, K. (2003), 'Pricing bounds on Asian options', Insurance: Mathematics \& Economics 38(2), 51-90.

Rao, C. (1997), Statistics and Truth: Putting Chance to Work, World Scientific.

Simon, S., Goovaerts, M. and Dhaene, J. (2000), 'An easy computable upper bound for the price of an arithmetic Asian option', Insurance: Mathematics \& Economics 26(2), 175-183.

Vanmaele, M., Deelstra, G., Liinev, J., Dhaene, J. and Goovaerts, M. (2006), 'Bounds for the price of discrete arithmetic asian options', Journal of Computational and Applied Mathematics 185(1), $51-90$.

Weber, A. and Wystup, U. (2009), Pricing formulae for foreign exchange options bibtex, in 'Encyclopedia of Quantitative Finance, Wiley'.

Zaks, Y., Frostig, E. and Levikson, B. (2006), 'Optimal pricing of a heterogeneous portfolio for a given risk level', ASTIN Bulletin 36, 161-185. 